(C) 5 International Press

Adv. Theor. Math. Phys. 2001 (5) 563-615

\title{
Standard-model bundles
}

\author{
Ron Donagi ${ }^{1}$, Burt A. Ovrut ${ }^{2}$, Tony Pantev ${ }^{1}$ and Daniel \\ Waldram ${ }^{3}$
}

${ }^{1}$ Department of Mathematics, University of Pennsylvania Philadelphia, PA 19104-6395, USA

${ }^{2}$ Department of Physics, University of Pennsylvania

Philadelphia, PA 19104-6396, USA

${ }^{3}$ Theory Division, CERN CH-1211, Geneva 23, Switzerland, and Department of Physics, The Rockfeller University

New York, NY 10021

\begin{abstract}
We describe a family of genus one fibered Calabi-Yau threefolds with fundamental group $\mathbb{Z} / 2$. On each Calabi-Yau $Z$ in the family we exhibit a positive dimensional family of Mumford stable bundles whose symmetry group is the Standard Model group $S U(3) \times S U(2) \times U(1)$ and which have $c_{3}=6$. We also show that for each bundle $V$ in our family, $c_{2}(Z)-c_{2}(V)$ is the class of an effective curve on $Z$. These conditions ensure that $Z$ and $V$ can be used for a phenomenologically relevant compactification of Heterotic M-theory.
\end{abstract}

MSC 2000: 14D21, 14J3

CERN-TH/2000-203, UPR-894T, RU-00-5B

e-print archive: http://xxx.lanl.gov/math.AG/0008010 


\section{Introduction}

In this paper we construct a particular class of bundles with constrained Chern classes on certain non-simply connected Calabi-Yau threefolds. These bundles are instrumental in deriving the Standard Model of particle physics in the context of the Heterotic M-theory [DOPWb]. Bundles of this type have been the subject of active research for quite some time [TY87], [Kac95], [PR99], [ACK], [DOPWc], [Tho]. In contrast with the classical constructions [TY87], [Kac95], [PR99], where the bundles obtained are associated with the tangent bundle of the Calabi-Yau manifold and tend to be rigid, our examples are independent of the geometry of the tangent bundle and vary in families. In particular we construct infinitely many positive dimensional families of bundles which are suitable for phenomenologically relevant compactifications of Heterotic M-theory. Our construction takes place entirely within the realm of algebraic geometry. The physical implications of our results are discussed in the companion paper [DOPWb], which also contains a summary of the construction written for physicists. In the remainder of this introduction we give a brief overview of the physical motivation for our work, followed by an outline of the actual geometric construction.

The search for exotic principal bundles on Calabi-Yau threefolds is motivated by string theory. To compactify the $E_{8} \times E_{8}$-Heterotic string to four dimensions one prescribes:

- a Calabi-Yau 3-fold $Z$;

- a Ricci flat Kähler metric on $Z$ with a Kähler form $\omega$;

- an $\omega$-instanton $\mathcal{E} \rightarrow Z$ with a structure group $E_{8} \times E_{8}$.

The Hermit-Einstein connection on $\mathcal{E}$ is a vacuum of the Heterotic string theory. The moduli space of $\mathcal{E}$ 's is a subspace of of the moduli space of vacua for the Heterotic string. In view of the Uhlenbeck-Yau theorem [UY86] every such $\mathcal{E}$ can be identified with an algebraic $E_{8} \times E_{8}$-bundle on $Z$ which is Mumford polystable with respect to the polarization $\omega$. In view of this theorem one can use algebraic geometry to study the moduli space of Heterotic vacua.

The type of bundles $\mathcal{E}$ allowed in a Heterotic compactification is restricted in physics in three ways:

(Supersymmetry preservation) $\mathcal{E}$ has to be Mumford polystable. 
(Anomaly cancellation) $c_{2}(\mathcal{E})=c_{2}(Z)$.

(Gauge symmetries) If the compactification of the Heterotic string has a group of symmetries $G \subset E_{8} \times E_{8}$, then the structure group of $\mathcal{E}$ can be reduced to the centralizer $G^{\prime}$ of $G$ in $E_{8} \times E_{8}$. Furthermore the corresponding $G^{\prime}$ bundle $\mathcal{E}_{G^{\prime}} \rightarrow Z$ should also be supersymmetric and anomaly-free.

Using these three principles one can look for special compactifications of the Heterotic string that reproduce in their low energy limits well understood and experimentally confirmed quantum field theories. Of particular interest are compactifications that will lead to the Standard Model of particle physics. For such compactifications one imposes two additional requirements on the triple $(Z, \mathcal{E}, \omega)$ :

(Standard Model gauge symmetries) The group $G$ of symmetries of $\mathcal{E}$, i.e. the centralizer inside $E_{8} \times E_{8}$ of a minimal subgroup $G^{\prime} \subset E_{8} \times E_{8}$ to which the structure group of $\mathcal{E}$ reduces, is $G=U(1) \times S U(2) \times S U(3)$.

\section{(3-generations condition)}

$$
\chi(\operatorname{ad}(\mathcal{E}))=\frac{c_{3}(\operatorname{ad}(\mathcal{E}))}{2}=3 .
$$

Triples $(Z, \omega, \mathcal{E})$ satisfying the five conditions above are hard to come by and tend to be rigid (see e.g. [Kac95]). However the recent advances in string theory prompted by the ground breaking work of Hořava-Witten [HW96b, HW96a, Wit96] on orbifold compactifications of M-theory, allow for a significant relaxation of the anomaly cancellation condition. This leads to two essential simplifications. First, it turns out that using the Horava-Witten mechanisms one can suppress completely one copy of $E_{8}$ in the structure group of $\mathcal{E}$. Secondly it was argued in [DLOW99, DOW99, ACK] that one can use M-theory 5-branes to relax the equality in the anomaly cancellation condition to an inequality. This leads to the following purely mathematical problem.

Main Problem. Find a smooth Calabi-Yau 3-fold $(Z, \omega)$ and a reductive subgroup $G^{\prime} \subset E_{8}$ so that

$\diamond$ the centralizer $G$ of $G^{\prime}$ in $E_{8}$ is a group isogenous to $S U(3) \times S U(2) \times$ $U(1)$; 
$\diamond$ there exists an $\omega$-stable $G_{\mathbb{C}}^{\prime}$-bundle $\mathcal{V} \rightarrow Z$ so that

- $c_{1}(\mathcal{V})=0$,

- $c_{2}(Z)-c_{2}(\mathcal{V})$ is the class of an effective reduced curve on $Z$,

- $c_{3}(\mathcal{V})=6$.

Here the Chern classes of $\mathcal{V}$ are calculated in the adjoint representation of $E_{8}$ considered as a representation of $G^{\prime}$. In fact for the physics applications it suffices for $G$ to contain a group isogenous to $S U(3) \times S U(2) \times U(1)$ as a direct summand.

The groups $G^{\prime} \subset E_{8}$ whose centralizer contains $S U(3) \times S U(2) \times U(1)$ as a direct summand can be classified. It turns out that there are no connected subgroups $G^{\prime}$ with $Z_{E_{8}}\left(G^{\prime}\right)=S U(3) \times S U(2) \times U(1)$. The stability assumption on $\mathcal{V}$ guarantees that the the structure group of $\mathcal{V}$ can not be reduced to a proper subgroup of $G_{\mathbb{C}}^{\prime}$. Therefore the structure group of the associated $\pi_{0}\left(G_{\mathbb{C}}^{\prime}\right)$-bundle $\mathcal{V} \times_{G_{\mathbb{C}}^{\prime}} \pi_{0}\left(G_{\mathbb{C}}^{\prime}\right)$ can not be reduced to a proper subgroup of $\pi_{0}\left(G_{\mathbb{C}}^{\prime}\right)$. Since $\mathcal{V} \times_{G_{\mathbb{C}}^{\prime}} \pi_{0}\left(G_{\mathbb{C}}^{\prime}\right)$ is a Galois cover of $Z$ with Galois group $\pi_{0}\left(G_{\mathbb{C}}^{\prime}\right)$, this just means that there should be a surjective homomorphism $\pi_{1}(Z) \rightarrow \pi_{0}\left(G_{\mathbb{C}}^{\prime}\right)$ and so we are forced to work with a non-simply connected $Z$.

Some possible choices for $G^{\prime}$ are: $S U(3) \times(\mathbb{Z} / 6), S U(4) \times(\mathbb{Z} / 3)$ and $S U(5) \times(\mathbb{Z} / 2)$. The corresponding centralizers are isogenous to $(S U(3) \times$ $S U(2) \times U(1)) \times U(1) \times U(1),(S U(3) \times S U(2) \times U(1)) \times U(1)$ and $S U(3) \times$ $S U(2) \times U(1)$. When $G_{0}^{\prime}$, the connected component of the identity in $G^{\prime}$, is a classical group, it turns out that the Chern classes of $\mathcal{V}$ in the fundamental representation of $G_{0}^{\prime}$ coincide with the Chern classes of $\mathcal{V}$ in the adjoint representation of $E_{8}$.

In this paper we explain how to build a big family of solutions of the Main Problem above for $G^{\prime}=S U(5) \times(\mathbb{Z} / 2)$.

For concreteness we look for $Z$ 's with $\pi_{1}(Z)=\mathbb{Z} / 2$. Let $\mathcal{V}$ be an $S L(5, \mathbb{C}) \times(\mathbb{Z} / 2)$-bundle on such a $Z$. Then $\mathcal{V}$ splits as a product of a rank five vector bundle and the unique non-trivial local system on $Z$ with monodromy $\mathbb{Z} / 2$. Pulling back this vector bundle to the universal cover $X$ of $Z$ we get a rank five vector bundle on $X$ which is invariant under the action of $\pi_{1}(Z)$ on $X$. Conversely every $\pi_{1}(Z)$-equivariant vector bundle $V \rightarrow X$ descends to a vector bundle on $Z$. Thus, in order to solve the Main 
Problem, it suffices to construct a quadruple $\left(X, \tau_{X}, H, V\right)$ such that the following conditions hold:

$(\mathbb{Z} / 2) X$ is a smooth Calabi-Yau 3-fold and $\tau_{X}: X \rightarrow X$ is a freely acting involution. $H$ is a fixed ample line bundle (Kähler structure) on $X$.

(S) $V$ is an $H$-stable vector bundle of rank five on $X$.

(I) $V$ is $\tau_{X}$-invariant.

(C1) $c_{1}(V)=0$.

(C2) $c_{2}(X)-c_{2}(V)$ is effective.

(C3) $c_{3}(V)=12$.

Since we need a mechanism for constructing bundles on $X$, we will choose $X$ to be elliptically fibered and use the so called spectral construction [FMW97, FMW99, Don97, BJPS97] to produce bundles on $X$. Note that the spectral construction applies only to elliptic fibrations, i.e. genus one fibrations with a section. This is the reason we build an equivariant $V$ on $X$ rather than obtaining directly $\mathcal{V}$ on $Z$. In general, there are two ways in which the spectral construction can be modified to work on genus one fibrations such as $Z$. One is to work with a spectral cover in the Jacobian fibration of $Z$ and an abelian gerbe on it. The other route (which is the one we chose) is to work with equivariant spectral data on the universal cover of $Z$. Note that there are higher algebraic structures involved in both approaches: the stackiness of the first approach is paralleled by complicated group actions on the derived category in the second.

More specifically we take $X$ to be a Calabi-Yau of Schoen type [Sch88], i.e. a fiber product of two rational elliptic surfaces $B$ and $B^{\prime}$ over $\mathbb{P}^{1}$, both in the four dimensional family described in [DOPWa]. The rank five bundle $V$ is built as an extension of two vector bundles $V_{2}$ and $V_{3}$ of ranks two and three respectively. Each of these is manufactured by the spectral construction. Alternatively $V$ may be viewed as a bundle corresponding to spectral data with a reducible spectral cover. Our preliminary research of this problem (some of which is recorded in [DOPWc]) showed that bundles corresponding to smooth spectral covers are unlikely to satisfy all of the above conditions. In fact, for the Calabi-Yau's we consider, one can show 
rigorously (see Remark 2.3) that $V$ 's coming from smooth spectral covers can never satisfy (I), (C1) and (C3) at the same time.

The paper is organized as follows. Section 2 describes the construction of $X$ and lists the geometric constraints on the spectral data which will ensure the validity of (I). Section 3 deals with the actual construction. We describe $V_{2}$ and $V_{3}$ in terms of their spectral data. The data for each $V_{i}$ involves a spectral curve $C_{i}$ in the surface $B$, a line bundle $\mathcal{N}_{i}$ on $C_{i}$, another line bundle $L_{i}$ on the surface $B^{\prime}$, and some optional parameters. The effect of taking these additional parameters to be non-zero is interpretted in section 3.2 as a series of Hecke transforms. The freedom to perform these Hecke transforms gives us at the end of the day infinitely many families of bundles. In section 4 we explain how the geometric information about the action of the spectral involution, obtained in [DOPWa, Theorem 7.1], takes care of condition (I). A delicate point here is that we need two genericity assumptions on $C_{i}$. The first one is that $C_{i}$ is finite over the base of the elliptic fibration on $B$. The second assumption is that $\operatorname{im}\left[\operatorname{Pic}(B) \rightarrow \operatorname{Pic}\left(C_{i}\right)\right]$ is Zariski dense in $\operatorname{Pic}^{0}\left(C_{i}\right)$. In sections 4.2 and 4.3 we check these two assumptions in the special case that is untimately utilized in the construction of $V$. In section 5 we translate the remaining conditions into a sequence of rather tight numerical inequalities. In Section 5.4 we show how the latter can be solved. In Section 6 we summarize the construction of $\left(X, \tau_{X}, H, V\right)$ and give an estimate on the dimension of the moduli space of such quadruples. Finally in Appendix A we have gathered some basic facts on Hecke transforms of vector bundles which are used in Section 3 .

Acknowledgements: We would like to thank Ed Witten, Dima Orlov, and Richard Thomas for valuable conversations on the subject of this work.

R. Donagi is supported in part by an NSF grant DMS-9802456 as well as a UPenn Research Foundation Grant. B. A. Ovrut is supported in part by a Senior Alexander von Humboldt Award, by the DOE under contract No. DE-AC02-76-ER-03071 and by a University of Pennsylvania Research Foundation Grant. T. Pantev is supported in part by an NSF grant DMS9800790 and by an Alfred P. Sloan Research Fellowship. D. Waldram would like to thank Enrico Fermi Institute at The University of Chicago and the Physics Department of The Rockefeller University for hospitality during the completion of this work. 


\section{Contents}

1 Introduction $\quad 564$

2 Elliptic Calabi-Yau threefolds with free involutions $\quad \mathbf{5 7 0}$

3 The construction $\quad 573$

3.1 The basic construction . . . . . . . . . . . . . . . . 573

3.2 Reinterpretation via Hecke transforms . . . . . . . . . 575

4 Invariant spectral data $\quad \mathbf{5 7 9}$

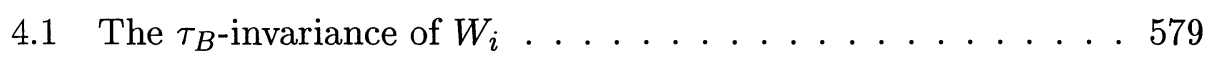

4.2 Invariance for $k_{2}=3 \ldots \ldots \ldots \ldots 83$

4.3 Invariance for $k_{3}=6 \ldots \ldots \ldots \ldots 55$

$\begin{array}{lll}5 & \text { Numerical conditions } & 587\end{array}$

5.1 The Chern classes of $V \ldots \ldots \ldots \ldots \ldots$. . . . . . . . 589

5.2 Stability of $V \ldots \ldots \ldots \ldots \ldots \ldots \ldots$

5.3 The list of constraints . . . . . . . . . . . . 5 595

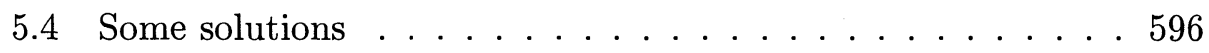

6 Summary of the construction $\quad 604$

6.1 The construction of $\left(X, \tau_{X}\right) \ldots \ldots \ldots 64$

6.2 The construction of $V \ldots \ldots \ldots \ldots 5$

$\begin{array}{ll}\text { Appendix A Hecke transforms } & 607\end{array}$

A.1 Definition and basic properties . . . . . . . . . 607

A.2 Geometric interpretation - flips . . . . . . . . . . 611

A.3 An example . . . . . . . . . . . . . . . . . 612 


\section{Elliptic Calabi-Yau threefolds with free involu- tions}

Our goal is to construct special $S U(5)$ bundles on smooth Calabi-Yau 3folds with fundamental group $\mathbb{Z} / 2$. We construct our Calabi-Yau 3-fold $Z$ as the quotient of a smooth Calabi-Yau 3-fold $X$ by a freely acting involution $\tau_{X}: X \rightarrow X$. Our $X$ will be elliptic and the elliptic fibration will be preserved by $\tau_{X}$, so that $Z$ will still have a genus one fibration. This enables us to apply the spectral construction to produce bundles.

The manifold $X$ is constructed as the fiber product $B \times_{\mathbb{P}^{1}} B^{\prime}$ of two rational elliptic surfaces $B$ and $B^{\prime}$ which live in the four dimensional family described in [DOPWa, Section 4]. For the first surface $B$ we use the notation from [DOPWa]. In particular we have $\beta: B \rightarrow \mathbb{P}^{1}, e, \zeta: \mathbb{P}^{1} \rightarrow B$ and the involutions $\alpha_{B}, \tau_{B}: B \rightarrow B$ and $\tau_{\mathbb{P}^{1}}: \mathbb{P}^{1} \rightarrow \mathbb{P}^{1}$. We use the same symbols with primes for the corresponding objects on $B^{\prime}$.

We choose an isomorphism of $\mathbb{P}^{1}$ with $\mathbb{P}^{1}$ which identifies $\tau_{\mathbb{P}^{1}}$ with $\tau_{\mathbb{P}^{1}}$ and sends $0 \in \mathbb{P}^{1}$ to $\infty^{\prime} \in \mathbb{P}^{1^{\prime}}$ and $\infty \in \mathbb{P}^{1}$ to $0^{\prime} \in \mathbb{P}^{1^{\prime}}$. With this convention we will make no distinction between $\mathbb{P}^{1}$ and $\mathbb{P}^{1^{\prime}}$ from now on.

Define $X:=B \times_{\mathbb{P}^{1}} B^{\prime}$. For a generic choice of $B$ and $B^{\prime}$ this $X$ will be smooth. It is an elliptic 3-fold in two ways: via its projections $\pi: X \rightarrow$ $B^{\prime}$ and $\pi^{\prime}: X \rightarrow B$. Since most of our analysis will involve the elliptic fibers we will work with the elliptic structure $\pi: X \rightarrow B^{\prime}$ in order to avoid cumbersome notation. By construction the discriminant of $\pi$ is in the linear system $\beta^{\prime *} \mathcal{O}_{\mathbb{P}^{1}}(12)=-12 K_{B^{\prime}}$ and so $X$ is a Calabi-Yau 3-fold.

For the zero section of $\pi$ we take the section $\sigma: B^{\prime} \rightarrow X$ corresponding to $e: \mathbb{P}^{1} \rightarrow B$. Let $\alpha_{X}:=\alpha_{B} \times_{\mathbb{P}^{1}} \tau_{B^{\prime}}$ and let $\tau_{X}:=\tau_{B} \times_{\mathbb{P}^{1}} \tau_{B^{\prime}}$. Since the fixed points of $\tau_{B}$ and $\tau_{B^{\prime}}$ sit over $\infty$ and 0 respectively, we conclude that $\tau_{X}$ acts freely on $X$. In particular the quotient $Z:=X / \tau_{X}$ is non-singular. We claim that $Z$ is in fact a Calabi-Yau. This is equivalent to saying that $\tau_{X}$ preserves the holomorphic 3-form on $X$. Indeed, $\tau_{X}$ acts on $H^{0}\left(X, \Omega_{X}^{3}\right)$ as multiplication by a number $\lambda \in \mathbb{C}^{\times}$. Since the fiber $f_{0} \times f_{0}^{\prime}$ of $X \rightarrow \mathbb{P}^{1}$ is stable under $\tau_{X}$ it suffices to compute the action of $\tau_{X}$ on $H^{0}\left(f_{0} \times f_{0}^{\prime}, \Omega_{X}^{3}\right)$. But

$$
\Omega_{X \mid f_{0} \times f_{0}^{\prime}}^{3}=\left(K_{f_{0}} \otimes K_{f_{0}^{\prime}}\right) \otimes T_{0}^{*} \mathbb{P}^{1}
$$

and $\tau_{X}$ acts as $\tau_{B \mid f_{0}}$ on $K_{f_{0}}, \tau_{B^{\prime} \mid f_{0}^{\prime}}$ on $K_{f_{0}^{\prime}}$ and as $\tau_{\mathbb{P}^{1}}$ on $T_{0}^{*} \mathbb{P}^{1}$. Since $\tau_{B \mid f_{0}}$ is a translation on $f_{0}$, it acts on $H^{0}\left(f_{0}, K_{f_{0}}\right)$ as +1 . Since $\tau_{B^{\prime} \mid f_{0}^{\prime}}$ and $\tau_{\mathbb{P}^{1}}$ each have a fixed point, they act as -1 on $H^{0}\left(f_{0}^{\prime}, K_{f_{0}^{\prime}}\right)$ and $T_{0}^{*} \mathbb{P}^{1}$ respectively. 
Hence $\lambda=1 \cdot(-1) \cdot(-1)=1$.

The vector bundles on $Z$ can be interpreted as $\tau_{X}$-invariant vector bundles on $X$. To construct vector bundles on $X$ we will exploit the fact that $X$ is an elliptically fibered 3 -fold and so we can manufacture bundles by using a relative Fourier-Mukai transform.

Concretely, let $\mathcal{P}_{X} \rightarrow X \times{ }_{B^{\prime}} X$ be the Poincare sheaf corresponding to the section $\sigma$. That is $\mathcal{P}_{X}$ is the rank one torsion-free sheaf given by

$$
\mathcal{P}_{X}=\mathcal{O}_{X \times_{B^{\prime}} X}\left(\Delta-\sigma \times_{B^{\prime}} X-X \times_{B^{\prime}} \sigma-m^{*} c_{1}\left(B^{\prime}\right)\right)=p_{13}^{*} \mathcal{P}_{B},
$$

where $m: X \times_{B^{\prime}} X \rightarrow B^{\prime}$ and $p_{13}: X \times_{B^{\prime}} X=B \times_{\mathbb{P}^{1}} B^{\prime} \times_{\mathbb{P}^{1}} B \rightarrow B \times_{\mathbb{P}^{1}} B$ are the natural projections. As in [DOPWa, Section 6], one argues that $\mathcal{P}_{X}$ defines an autoequivalence (see [BM, Theorem 1.2]) of $D^{b}(X)$ :

$$
\begin{aligned}
\boldsymbol{F} \boldsymbol{M}_{X}: \quad D^{b}(X) & \longrightarrow D^{b}(X) \\
\mathcal{F} & \longmapsto R^{\bullet} p_{1 *}\left(p_{2}^{*} \mathcal{F} \stackrel{L}{\otimes} \mathcal{P}_{X}\right) .
\end{aligned}
$$

If $V \rightarrow X$ is a vector bundle of rank $r$ which is semistable and of degree zero on each fiber of $\pi: X \rightarrow B^{\prime}$, then its Fourier-Mukai transform $\boldsymbol{F} \boldsymbol{M}_{X}(V)[1]$ is a torsion sheaf of pure dimension two on $X$. The support of $\boldsymbol{F} \boldsymbol{M}_{X}(V)[1]$ is a surface $i_{\Sigma}: \Sigma \hookrightarrow X$ which is finite of degree $r$ over $B^{\prime}$. Furthermore $\boldsymbol{F} \boldsymbol{M}_{X}(V)$ is of rank one on $\Sigma$. In fact, if $\Sigma$ is smooth, then $\boldsymbol{F} \boldsymbol{M}_{X}(V)[1]=i_{\Sigma *} L$ is just the extension by zero of some line bundle $L \in \operatorname{Pic}(\Sigma)$. Conversely if $\mathcal{N} \rightarrow X$ is a sheaf of pure dimension two which is flat over $B^{\prime}$, then $\boldsymbol{F} \boldsymbol{M}_{X}(\mathcal{N})$ is a vector bundle on $X$ of rank equal to the degree of $\operatorname{supp}(\mathcal{N})$ over $B^{\prime}$ and whose first Chern class is vertical (for the projection $\pi: X \rightarrow B^{\prime}$ ). This correspondence between vector bundles on $X$ and sheaves on $X$ supported on finite covers of $B^{\prime}$ is commonly known as the spectral construction and has been extensively studied in the context of Weierstrass elliptic fibrations [FMW97, FMW99, Don97, BJPS97]. The torsion sheaf $\mathcal{N}$ on $X$ is called spectral datum and the surface $\Sigma=\operatorname{supp} \mathcal{N}$ is called a spectral cover.

Since our elliptic Calabi-Yau $X$ is not Weierstrass we briefly describe how the spectral construction works (at least for generic spectral data) on $X$ and how it interacts with the involution $\tau_{X}$. First we need to understand the action of $\boldsymbol{F} \boldsymbol{M}_{X}$ on line bundles on $X$. Note that since $X=B \times_{\mathbb{P}^{1}} B^{\prime}$ is a fiber product we have $\operatorname{Pic}(X)=\left(\operatorname{Pic}(B) \times \operatorname{Pic}\left(B^{\prime}\right)\right) / \operatorname{Pic}\left(\mathbb{P}^{1}\right)$. In particular, every line bundle on $X$ can be written as $L \otimes L^{\prime}:=\pi^{*} L \otimes \pi^{*} L^{\prime}$ for some $L \rightarrow B$ and $L^{\prime} \rightarrow B^{\prime}$.

Lemma 2.1. For every line bundle $\mathcal{L}=L \otimes L^{\prime}$ on $X$, the actions of the Fourier-Mukai transform and of the spectral involution are given by: 
(a) $\boldsymbol{F} \boldsymbol{M}_{X}(\mathcal{L})=\boldsymbol{F} \boldsymbol{M}_{X}\left(L \otimes L^{\prime}\right)=\pi^{\prime}{ }^{*} \boldsymbol{F} \boldsymbol{M}_{B}(L) \otimes \pi^{*} L^{\prime}=\boldsymbol{F} \boldsymbol{M}_{B}(L) \otimes L^{\prime}$.

(b) $\boldsymbol{T}_{X}(\mathcal{L}):=\left(\boldsymbol{F} \boldsymbol{M}_{X}^{-1} \circ \tau_{X}^{*} \circ \boldsymbol{F} \boldsymbol{M}_{X}\right)(\mathcal{L})=\pi^{*}\left(\boldsymbol{T}_{B}(L)\right) \otimes \pi^{*}\left(\tau_{B^{\prime}}^{*} L^{\prime}\right)=$ $\boldsymbol{T}_{B}(L) \otimes \tau_{B^{\prime}}^{*} L^{\prime}$.

Proof. Part (b) is an obvious consequence of part (a). To prove part (a) we will use the identification $X \times_{B^{\prime}} X=B \times_{\mathbb{P}^{1}} B^{\prime} \times_{\mathbb{P}^{1}} B$. In terms of this identification we have:

$$
\begin{aligned}
\boldsymbol{F} \boldsymbol{M}_{X}(\mathcal{L}) & =R p_{23 *}\left(p_{12}^{*} \mathcal{L} \otimes \mathcal{P}_{X}\right) \\
& =R p_{23 *}\left(p_{12}^{*}\left(L \otimes L^{\prime}\right) \otimes p_{13}^{*} \mathcal{P}_{B}\right) \\
& =R p_{23 *}\left(\pi_{1}^{*} L \otimes \pi_{2}^{*} L^{\prime} \otimes p_{13}^{*} \mathcal{P}_{B}\right) \\
& =R p_{23 *}\left(p_{13}^{*}\left(p_{1}^{*} L \otimes \mathcal{P}_{B}\right) \otimes p_{23}^{*}\left(\pi^{*} L^{\prime}\right)\right) \\
& =R p_{23 *}\left(p_{13}^{*}\left(p_{1}^{*} L \otimes \mathcal{P}_{B}\right)\right) \otimes \pi^{*} L^{\prime}
\end{aligned}
$$

Here $\pi_{1}, \pi_{2}$ and $\pi_{3}$ are the natural projections of $B \times_{\mathbb{P}^{1}} B^{\prime} \times_{\mathbb{P}^{1}} B$ onto $B, B^{\prime}$ and $B$ respectively, $p_{i}: B \times_{\mathbb{P}^{1}} B \rightarrow B$ are the projections on the two factors, and in the last identity we have used the projection formula for $p_{23}$.

Now using the base change property for the fiber square

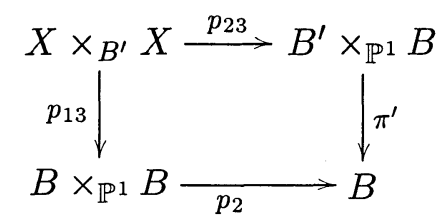

we get $R p_{23 *} p_{13}^{*}=\pi^{\prime *} R p_{2 *}$ and so

$$
\boldsymbol{F} \boldsymbol{M}_{X}(\mathcal{L})=\pi^{*}\left(R p_{2 *}\left(p_{1}^{*} L \otimes \mathcal{P}_{B}\right)\right) \otimes \pi^{*} L^{\prime}=\boldsymbol{F} \boldsymbol{M}_{B}(L) \otimes L^{\prime} .
$$

The lemma is proven.

Let now $i_{\Sigma}: \Sigma \hookrightarrow X$ be a surface which is finite and of degree $r$ over $B^{\prime}$. Then for any line bundle $\mathcal{L} \in \operatorname{Pic}(X)$ the torsion sheaf $\mathcal{N}:=i_{\Sigma *} i_{\Sigma}^{*} \mathcal{L}$ has a resolution by global line bundles. Namely

$$
0 \rightarrow \mathcal{L}(-\Sigma) \rightarrow \mathcal{L} \rightarrow \mathcal{N} \rightarrow 0
$$

In particular $\mathcal{N}$ is quasi-isomorphic to the two-step complex of line bundles $[\mathcal{L}(-\Sigma) \rightarrow \mathcal{L}]$ on $X$ and so the actions of $\boldsymbol{F} \boldsymbol{M}_{X}$ and $\boldsymbol{T}_{X}$ on $\mathcal{N}$ can be computed via the formulas in Lemma 2.1. Specifically we have:

Lemma 2.2. Let $\mathcal{L}=L \otimes L^{\prime}$ be a global line bundle on $X$ and let $i_{\Sigma}: \Sigma \hookrightarrow X$ be a surface finite over $B^{\prime}$. Let $\mathcal{N}=i_{\Sigma *} i_{\Sigma}^{*} \mathcal{L}$ be such that $V=\boldsymbol{F} \boldsymbol{M}_{X}(\mathcal{N})$ is a rank $r$ vector bundle on $X$ with $c_{1}(V)=0$. Then $\tau_{X}^{*} V \cong V$ if and only if the following three conditions 
- $\alpha_{X}(\Sigma)=\Sigma$;

- $\tau_{B^{\prime}}^{*} L^{\prime} \cong L^{\prime}$;

- $\boldsymbol{T}_{B}^{*} L \cong L$.

are satisfied.

Remark 2.3. Notice that the $\tau_{X}$ invariance of $V$ amounts to two separate conditions on the spectral data. The first is that the spectral surface $\Sigma$ has to be invariant under the involution $\alpha_{X}$. This condition is relatively easy to satisfy. It just means that $\Sigma$ is pulled back from the quotient $X / \alpha_{X}$. The second condition requires the $\tau_{B^{\prime}}$ invariance of $L^{\prime}$ and the $\boldsymbol{T}_{B}$ invariance of $L$.

In fact, the formulas in [DOPWa, Table 3] (written in terms of the basis of $H^{2}(B, \mathbb{Z})$ described in $[D O P W a$, Section 4.2]) show that $L \in \operatorname{Pic}(B) \otimes \mathbb{Q}$ will be $\boldsymbol{T}_{B}$-invariant if and only if $L$ is in the affine subspace

$-\frac{1}{2} e_{1}+\operatorname{Span}_{\mathbb{Q}}\left(f, e_{9}, e_{4}-e_{5}, e_{4}-e_{6}, 3 \ell-2\left(e_{4}+e_{5}+e_{6}\right)-3 e_{7}, \ell-e_{7}-2 e_{8}\right)$,

which does not intersect $\operatorname{Pic}(B) \subset \operatorname{Pic}(B) \otimes \mathbb{Q}$. This implies that $V$ can not be $\tau_{X}$-invariant if $\mathcal{N}=i_{\Sigma *} i_{\Sigma}^{*} \mathcal{L}$ for some global $\mathcal{L} \in \operatorname{Pic}(X)$. For $\Sigma$ smooth and very ample the Lefschetz hyperplane section theorem asserts that every $\mathcal{N}$ comes from a global $\mathcal{L}$ and hence one is forced to work with singular or non-very ample surfaces $\Sigma$.

\section{The construction}

\subsection{The basic construction}

In this section we describe in detail our method of constructing $\tau_{X}$-invariant vector bundles on $X$.

In order to circumvent the difficulty pointed out in Remark 2.3 we build our rank five bundle $V$ on $X$ not directly by the spectral construction but as an extension

$$
0 \rightarrow V_{2} \rightarrow V \rightarrow V_{3} \rightarrow 0
$$


Here $V_{i}, i=2,3$ is a rank $i$ bundle on $X$ which is $\tau_{X}$-invariant and satisfies some strong numerical conditions which will be discussed in the next section. In addition, we will see that the stability condition on $V$ amounts to the extension being non split.

Each $V_{i}$ is produced by an application of the spectral construction on $X$ with a reducible spectral cover and a a line bundle on it which is not the restriction of a global line bundle on $X$. Define $V_{i}$ from its spectral data as follows:

- Let $C_{i}$ be a curve in the linear system $\left|\mathcal{O}_{B}\left(i e+k_{i} f\right)\right|$ where $k_{i}$ is an integer. Let $\Sigma_{i}:=C_{i} \times_{\mathbb{P}^{1}} B^{\prime}$. Recall that $\beta^{\prime}: B^{\prime} \rightarrow \mathbb{P}^{1}$ has two $I_{2}$ fibers $f_{1}^{\prime}, f_{2}^{\prime}$. Let $F_{j}, j=1,2$ be the corresponding fibers of $B$; note that, because of the way we glued the $\mathbb{P}^{1}$ bases, these are not the reducible fibers $f_{j}$ of $B$. Let

$$
\left\{p_{i j k}\right\}_{k=1}^{i}:=C_{i} \cap F_{j} .
$$

Then $\Sigma_{i} \rightarrow C_{i}$ is an elliptic surface having $2 i$ fibers of type $I_{2}:\left(n_{j}^{\prime} \cup\right.$ $\left.o_{j}^{\prime}\right) \times\left\{p_{i j k}\right\}$ where $j=1,2$ and $k=1, \ldots, i$.

- Define $V_{i}$ as

$V_{i}^{*}=\boldsymbol{F} \boldsymbol{M}_{X}\left(\left(\Sigma_{i},\left(\pi_{\mid \Sigma_{i}}^{\prime}\right)^{*} \mathcal{N}_{i} \otimes \pi^{*} L_{i} \otimes \mathcal{O}_{\Sigma_{i}}\left(-\sum\left\{p_{i j k}\right\} \times\left(a_{i j k} n_{j}^{\prime}+b_{i j k} o_{j}^{\prime}\right)\right)\right)\right)$,

where $L_{i}$ is a line bundle on $B^{\prime}, \mathcal{N}_{i}$ is a line bundle on the curve $C_{i}$ and the optional parameters $a_{i j k}, b_{i j k}$ are integers.

Note that there is a redundancy in our choices, because $n_{j}^{\prime}+o_{j}^{\prime}=f_{j}^{\prime}$ is a pullback from $\mathbb{P}^{1}$, and so can be absorbed in $L_{i}$. In particular, we can always arrange for all the coefficients $a_{i j k}, b_{i j k}$ to be non-negative. Also, without a loss of generality we may assume that for any given $j$ we have $a_{i j k} \cdot b_{i j k}=0$ for all $i, k$. With this convention, we have an alternative description of $V_{i}$ : Put

$$
\widetilde{W}_{i}:=V_{i} \otimes \pi^{*} L_{i}^{-1}
$$

It turns out that the bundle $\widetilde{W}_{i}$ can be constructed directly. Consider the vector bundle $W_{i}$ on $B$, built by the spectral construction as $W_{i}:=$ $\boldsymbol{F} \boldsymbol{M}_{B}\left(C_{i}, \mathcal{N}_{i}\right)$. Then $\widetilde{W}_{i}$ is obtained from the vector bundle $\pi^{*} W_{i}$ by $a_{i j k}$ successive Hecke transforms along the divisors $\left\{p_{i j k}\right\} \times n_{j}^{\prime}$ and $b_{i j k}$ successive Hecke transforms along the divisors $\left\{p_{i j k}\right\} \times o_{j}^{\prime}$. The center of each Hecke is 
a line bundle on the surface $F_{j} \times n_{j}^{\prime}$ or $F_{j} \times o_{j}^{\prime}$. (For the definition and basic properties of Hecke transforms see appendix A).

In fact, $V$ itself could be built by applying the spectral construction on $X$ to the reducible spectral cover $\Sigma_{2} \cup \Sigma_{3}$ and an appropriately chosen sheaf on it. However the construction with extensions is technically easier because it allows us to avoid dealing with sheaves on singular surfaces. This approach is a variation on the method employed by Richard Thomas in [Tho].

Remark 3.1. Observe that in the definition of $C_{i}$ we could have taken the linear system more generally to be of the form $\left|\mathcal{O}_{B}\left(i e+k_{i} f+\eta_{i}\right)\right|$ where $k_{i}$ is an integer and $\eta_{i} \in \operatorname{Pic}(B)$ is a class perpendicular to $e$ and $f$. If we impose the condition $c_{1}\left(W_{i}\right)=0$, then the classes $\eta_{i}$ are forced to be zero by the Riemann-Roch formula. However the introduction of the $L_{i}$ 's gives us the extra freedom of working with $W_{i}$ 's that have arbitrary vertical $c_{1}$. We will not exploit this extra freedom but we expect that many examples exist which are similar to ours but have $\eta_{i} \neq 0$.

Since the Hecke interpretation of $\widetilde{W}_{i}$ will be important in determining the invariance properties of $V$ and in implementing the numerical constraints, we proceed to spell it out explicitly.

\subsection{Reinterpretation via Hecke transforms}

Recall from section 2 that $X=B \times_{\mathbb{P}^{1}} B^{\prime}$ fits into a commutative diagram of projections

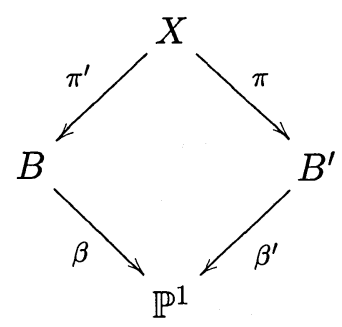

Let $C \subset B$ be a smooth connected curve in the linear system $\left|\mathcal{O}_{B}(r e+k f)\right|$. Let $\mathcal{N} \in \operatorname{Pic}^{d}(C)$ and let $W:=\boldsymbol{F M}_{B}((C, \mathcal{N}))$ be the corresponding rank $r$ vector bundle on $B$.

Consider $\Sigma=\pi^{\prime-1}(C)=C \times_{\mathbb{P}^{1}} B^{\prime}$ and the line bundle $\mathcal{L}=\left(\pi_{\mid \Sigma}^{\prime}\right)^{*} \mathcal{N}=$ $\mathcal{N} \otimes \mathcal{O}_{B^{\prime}}$ on $\Sigma$. 
Let $f_{j}^{\prime}=n_{j}^{\prime} \cup o_{j}^{\prime}, F_{j}=\beta^{-1}\left(\beta^{\prime}\left(f_{j}^{\prime}\right)\right), j=1,2$ be as above. We assume that $C$ is general enough so that the intersections $C \cap F_{j}, j=1,2$ are transversal.

Let $p \in C \cap F_{j}$ and let $a$ be a non-negative integer. Define

$$
W[a, p]:=\boldsymbol{F} \boldsymbol{M}_{X}\left(\left(\Sigma, \mathcal{L}\left(-a\left(\{p\} \times n_{j}^{\prime}\right)\right)\right)\right) .
$$

Consider the divisor $D=F_{j} \times n_{j}^{\prime} \subset X$ and the line bundles

$$
\mathcal{O}_{F_{j}}(p-e) \otimes \mathcal{O}_{n_{j}^{\prime}}(2 a) \in \operatorname{Pic}(D),
$$

where $a \in \mathbb{Z}$ and by a abuse of notation $e$ denotes the point of intersection of the curves $e, F_{j}$ in $B$. With this notation we have

Lemma 3.2. Fix $a \geq 0$.

(i) There is a canonical surjective map $W[a, p]_{\mid D} \rightarrow \mathcal{O}_{F_{j}}(p-e) \otimes \mathcal{O}_{n_{j}^{\prime}}(2 a)$ which fits in a short exact sequence of vector bundles on $D$

$$
\left(\psi_{a+1}\right) \quad 0 \rightarrow K_{a} \rightarrow W[a, p]_{\mid D} \rightarrow \mathcal{O}_{F_{j}}(p-e) \otimes \mathcal{O}_{n_{j}^{\prime}}(2 a) \rightarrow 0 .
$$

(ii) For $a=0$ we have $W[0, p]=\pi^{*} W$ and for $a \geq 1$

$$
W[a, p]=\operatorname{Hecke}_{\left(\psi_{a}\right)}^{-} \circ \operatorname{Hecke}_{\left(\psi_{a-1}\right)}^{-} \circ \ldots \circ \operatorname{Hecke}_{\left(\psi_{1}\right)}^{-}\left(\pi^{\prime *} W\right) .
$$

Proof. We will prove the lemma by induction in $a$. By definition we have $W[0, p]=\pi^{\prime} W$ which takes care of the base of the induction. Assume that $W[i, p]=\boldsymbol{H e c k e}_{\left(\psi_{i}\right)}^{-}(W[i-1, p])$ for all $0<i<a$. Consider the short exact sequence of sheaves on $\Sigma$ :

$$
\begin{aligned}
0 \rightarrow \mathcal{L}\left(-a\left(\{p\} \times n_{j}^{\prime}\right)\right) & \rightarrow \mathcal{L}\left(-(a-1)\left(\{p\} \times n_{j}^{\prime}\right)\right) \\
& \rightarrow \mathcal{L}\left(-(a-1)\left(\{p\} \times n_{j}^{\prime}\right)\right)_{\mid\{p\} \times n_{j}^{\prime}} \rightarrow 0 .
\end{aligned}
$$

We have $\mathcal{L}_{\mid\{p\} \times n_{j}^{\prime}}=\left(\left(\pi_{\mid \Sigma}^{\prime}\right)^{*} \mathcal{N}\right)_{\mid\{p\} \times n_{j}^{\prime}}=\mathcal{O}_{\{p\} \times n_{j}^{\prime}}$. Also $\{p\} \times n_{j}^{\prime}$ is a component of an $I_{2}$ fiber of the elliptic surface $\pi_{\mid \Sigma}^{\prime}: \Sigma \rightarrow C$ and so $\mathcal{O}_{\{p\} \times n_{j}^{\prime}}(\{p\} \times$ $\left.n_{j}^{\prime}\right)=\mathcal{O}_{\{p\} \times n_{j}^{\prime}}(-2)$. Let $i_{\Sigma}: \Sigma \hookrightarrow X$ and $\iota: n_{j}^{\prime}=\{p\} \times n_{j}^{\prime} \hookrightarrow \Sigma \subset X$ denote the natural inclusions. Then if we extend each of the sheaves in the sequence (3.2) by zero we obtain a short exact sequence of sheaves on $X$ :

$0 \rightarrow i_{\Sigma *} \mathcal{L}\left(-a\left(\{p\} \times n_{j}^{\prime}\right)\right) \rightarrow i_{\Sigma *} \mathcal{L}\left(-(a-1)\left(\{p\} \times n_{j}^{\prime}\right)\right) \rightarrow \iota_{*} \mathcal{O}_{n_{j}^{\prime}}(2(a-1)) \rightarrow 0$.

Applying $\boldsymbol{F} \boldsymbol{M}_{X}$ to (3.4) we get

$$
0 \rightarrow W[a, p] \rightarrow W[a-1, p] \rightarrow \boldsymbol{F} \boldsymbol{M}_{X}\left(\iota_{*} \mathcal{O}_{n_{j}^{\prime}}(2(a-1))\right) \rightarrow 0 .
$$


By the definition of $\boldsymbol{F} \boldsymbol{M}_{X}$ we have

$$
\boldsymbol{F} \boldsymbol{M}_{X}\left(\iota_{*} \mathcal{O}_{n_{j}^{\prime}}(2(a-1))\right)=R p_{2 *}^{\bullet}\left(p_{1}^{*}\left(\iota_{*} \mathcal{O}_{n_{j}^{\prime}}(2(a-1)) \stackrel{L}{\otimes} \mathcal{P}_{X}\right),\right.
$$

where $p_{1}, p_{2}: X \times_{B^{\prime}} X \rightarrow X$ are the natural projections.

If we use the identification $X \times_{B^{\prime}} X \cong B \times \times_{\mathbb{P}^{1}} B \times_{\mathbb{P}^{1}} B^{\prime}$, then the projection $p_{i}: X \times_{B^{\prime}} X \rightarrow X$ gets identified with the projection $p_{i 3}: B \times_{\mathbb{P}^{1}} B \times_{\mathbb{P}^{1}} B^{\prime} \rightarrow$ $B \times \times_{\mathbb{P}^{1}} B^{\prime}$ and $\mathcal{P}_{X}=p_{12}^{*} \mathcal{P}_{B}$. In particular in terms of the identification $X \times_{B^{\prime}} X \cong B \times_{\mathbb{P}^{1}} B \times_{\mathbb{P}^{1}} B^{\prime}$ we see that $p_{1}^{*}\left(\iota_{*} \mathcal{O}_{n_{j}^{\prime}}(2(a-1))\right)$ is supported on the surface $\{p\} \times F_{j} \times n_{j}^{\prime} \subset B \times \times_{\mathbb{P}^{1}} B \times_{\mathbb{P}^{1}} B^{\prime}$ is precisely

$$
\begin{aligned}
\operatorname{pr}_{n_{j}^{\prime}}^{*} \mathcal{O}_{n_{j}^{\prime}}(2(a-1)) \otimes \mathcal{P}_{X \mid\{p\} \times F_{j} \times n_{j}^{\prime}} & =\operatorname{pr}_{n_{j}^{\prime}}^{*} \mathcal{O}_{n_{j}^{\prime}}(2(a-1)) \otimes \operatorname{pr}_{F_{j}}^{*} \mathcal{P}_{B \mid\{p\} \times F_{j}} \\
& =\operatorname{pr}_{n_{j}^{\prime}}^{*} \mathcal{O}_{n_{j}^{\prime}}(2(a-1)) \otimes \operatorname{pr}_{F_{j}}^{*} \mathcal{O}_{n_{j}^{\prime}}(p-e) .
\end{aligned}
$$

Also for the restricted map $p_{2 \mid\{p\} \times F_{j} \times n_{j}^{\prime}}:\{p\} \times F_{j} \times n_{j}^{\prime} \rightarrow X$ we get

$$
p_{2 \mid\{p\} \times F_{j} \times n_{j}^{\prime}}=p_{23 \mid\{p\} \times F_{j} \times n_{j}^{\prime}}=i_{D}
$$

and hence

$$
\begin{aligned}
\boldsymbol{F} \boldsymbol{M}_{X}\left(\iota_{*} \mathcal{O}_{n_{j}^{\prime}}(2(a-1))\right) & =R_{p_{2 *}}^{\bullet}\left(p_{1}^{*}\left(\iota_{*} \mathcal{O}_{n_{j}^{\prime}}(2(a-1)) \stackrel{L}{\otimes} \mathcal{P}_{X}\right)\right. \\
& =i_{D *}\left(\mathcal{O}_{F_{j}}(p-e) \otimes \mathcal{O}_{n_{j}^{\prime}}(2(a-1))\right) .
\end{aligned}
$$

which combined with (3.5) concludes the proof of the lemma.

If now $b \geq 0$ is another integer we may consider also the vector bundle

$$
W\{b, p\}=\boldsymbol{F} \boldsymbol{M}_{X}\left(\left(\Sigma, \mathcal{L}\left(-b\left(\{p\} \times o_{j}^{\prime}\right)\right)\right)\right) .
$$

In exactly the same way we see that $W\{0\}=\pi^{\prime}{ }^{*} W$, that for every $b \geq 1$ there is a canonical exact sequence

$$
\left(\phi_{b+1}\right) \quad 0 \rightarrow M_{b} \rightarrow W\{b, p\}_{\mid\left\{F_{j}\right\} \times o_{j}^{\prime}} \rightarrow \mathcal{O}_{F_{j}}(p-e) \otimes \mathcal{O}_{o_{j}^{\prime}}(2 b) \rightarrow 0,
$$

and that

$$
W\{b, p\}=\operatorname{Hecke}_{\left(\phi_{a}\right)}^{-} \circ \operatorname{Hecke}_{\left(\phi_{a-1}\right)}^{-} \circ \ldots \circ \operatorname{Hecke}_{\left(\phi_{1}\right)}^{-}\left(\pi^{\prime *} W\right) .
$$

For future reference we record

Corollary 3.3. The Chern classes of $W[a, p]$ and $W\{b, p\}$ are given by

$$
\begin{aligned}
\operatorname{ch}(W[a, p]) & =\pi^{\prime} \operatorname{ch}(W)-a \pi^{*} n_{j}^{\prime}-a^{2}(f \times \mathrm{pt}) ; \\
\operatorname{ch}(W\{b, p\}) & =\pi^{*} \operatorname{ch}(W)-b \pi^{*} o_{j}^{\prime}-b^{2}(f \times \mathrm{pt})
\end{aligned}
$$


Proof. Clearly it suffices to prove the corollary for $W[a, p]$. By Lemma 3.2 we have short exact sequences

$$
0 \rightarrow W[n, p] \rightarrow W[n-1, p] \rightarrow i_{D *} \psi_{n} \rightarrow 0
$$

for all $n \geq 1$. Here we have slightly abused the notation by writing $\psi_{a}$ for the middle term of the short exact sequence $\left(\psi_{a}\right)$. Hence $\operatorname{ch}(W[n, p])=$ $\operatorname{ch}(W[n-1, p])-\operatorname{ch}\left(i_{D *} \psi_{n}\right)$ and so

$$
\operatorname{ch}(W[a, p])=\pi^{*} \operatorname{ch}(W)-\sum_{n=1}^{a} \operatorname{ch}\left(i_{D *} \psi_{n}\right) .
$$

Using Grothendieck-Riemann-Roch we calculate

$$
\begin{aligned}
\operatorname{ch}\left(i_{D *} \psi_{n}\right) & =i_{D *}\left(\operatorname{ch}\left(\psi_{n}\right) t d(D)\right) t d(X)^{-1} \\
& =i_{D *}\left(\left(1+\psi_{n}+\frac{\psi_{n}^{2}}{2}\right)\left(1+F_{j} \times \mathrm{pt}\right)\right)\left(1-\left(f \times \mathrm{pt}+\mathrm{pt} \times f^{\prime}\right)\right) \\
& =i_{D *}\left(\left(1+2(n-1) F_{j} \times \mathrm{pt}\right)\left(1+F_{j} \times \mathrm{pt}\right)\right)\left(1-\left(f \times \mathrm{pt}+\mathrm{pt} \times f^{\prime}\right)\right) \\
& =i_{D *}\left(1+(2 n-1) F_{j} \times \mathrm{pt}\right)\left(1-\left(f \times \mathrm{pt}+\mathrm{pt} \times f^{\prime}\right)\right) \\
& =D+(2 n-1)(f \times \mathrm{pt})=\pi^{*} n_{j}^{\prime}+(2 n-1)(f \times \mathrm{pt}) .
\end{aligned}
$$

Consequently

$$
\begin{aligned}
\operatorname{ch}(W[a, p]) & =\pi^{*} \operatorname{ch}(W)-\sum_{n=1}^{a}\left(\pi^{*} n_{j}^{\prime}+(2 n-1)(f \times \mathrm{pt})\right. \\
& =\pi^{*} \operatorname{ch}(W)-a \pi^{*} n_{j}^{\prime}-a^{2}(f \times \mathrm{pt}) .
\end{aligned}
$$

The corollary is proven.

Finally, we are ready to give the Hecke interpretation of $\widetilde{W}_{i}=V_{i} \otimes \pi^{*} L_{i}^{-1}$. Recall that

$$
\widetilde{W}_{i}=\boldsymbol{F} \boldsymbol{M}_{X}\left(\left(\Sigma_{i}, \mathcal{L}_{i}\left(-\sum\left\{p_{i j k}\right\} \times\left(a_{i j k} n_{j}^{\prime}+b_{i j k} o_{j}^{\prime}\right)\right)\right)\right)
$$

where $a_{i j k}, b_{i j k}$ are non-negative integers satisfying $a_{i j k} b_{i j k}=0$. Since Hecke transforms whose centers have disjoint supports obviously commute, we see from the above discussion that

$$
\widetilde{W}_{i}=W\left[a_{i 11}, p_{i 11}\right]\left\{b_{i 11}, p_{i 11}\right\}\left[a_{i 12}, p_{i 12}\right]\left\{b_{i 12}, p_{i 12}\right\} \ldots\left[a_{i 1 i}, p_{i 1 i}\right]\left\{b_{i 1 i}, p_{i 1 i}\right\} .
$$




\section{Invariant spectral data}

In this section we examine the conditions for $V$ to be $\tau_{X}$-invariant. It is easy to reduce this, first to invariance of the $V_{i}$, then to invariance of the $W_{i}$. Indeed, assume that the bundles $V_{i}$ are $\tau_{X}$-invariant, and choose liftings of the $\tau_{X}$ action to the $V_{i}$. The space $\operatorname{Ext}^{1}\left(V_{3}, V_{2}\right)$ parameterizing all extensions is a direct sum of its invariant and anti-invariant subspaces. So if $\operatorname{Ext}^{1}\left(V_{3}, V_{2}\right) \neq 0$ we also have an extension which is either invariant or anti-invariant. Finally, changing the lifted action of $\tau_{X}$ on one of the $V_{i}$ interchanges invariants with anti-invariants, so we are done.

Since $V_{i}=\widetilde{W}_{i} \otimes \pi^{*} L_{i}$, we have

$$
\tau_{X}^{*}\left(V_{i}\right)=\tau_{X}^{*}\left(\widetilde{W}_{i}\right) \otimes \pi^{*} \tau_{B^{\prime}}^{*} L_{i}
$$

So it suffices to have a $\widetilde{W}_{i}$ which is $\tau_{X}$-invariant and an $L_{i}$ which is $\tau_{B^{\prime}}$ invariant. From [DOPWa, Table 1] we know that there is a 6 -dimensional lattice of $\tau_{B^{\prime}}$-invariant classes on $B^{\prime}$, so we have lots of possibilities for the $L_{i}$. Now $\widetilde{W}_{i}$ is a Hecke transform of $\pi^{\prime *}\left(W_{i}\right)$, so we want $W_{i}$ to be $\tau_{B}$-invariant and the center of the Hecke transform to be $\tau_{X}$-invariant. Above we took the support of this Hecke to be an arbitrary collection of components of the surfaces $F_{j} \times f_{j}^{\prime}$ for $j=1,2$. It can be seen from [DOPWa, Table 1] together with the expression [DOPWa, Formula (4.2)] for the components of the $I_{2}$-fibers of $B$ in terms of our basis, that the action of $\tau_{B^{\prime}}$ interchanges $o_{1}$ with $n_{2}$ and $o_{2}$ with $n_{1}$. Therefore the condition for $\tau_{X}$-invariance of the center of the Hecke transform becomes $a_{i 1 k}=b_{i 2 k}$ and $a_{i 2 k}=b_{i 1 k}$. Because of the redundancy in our choices we are free to take $a_{i 2 k}=b_{i 1 k}=0$ and $a_{i 1 k}=b_{i 2 k} \geq 0$.

Finally we have to find the conditions that will ensure the $\tau_{B}$-invariance of $W_{i}$.

\subsection{The $\tau_{B}$-invariance of $W_{i}$}

Throughout this subsection we work with a spectral curve $C_{i}$ in the linear system $\left|i e+k_{i} f\right|, i=2$ or 3 , which is finite over $\mathbb{P}^{1}$, and a line bundle $\mathcal{N}_{i} \in \operatorname{Pic}\left(C_{i}\right)$. The $\tau_{B}$-invariance of $W_{i}=\boldsymbol{F} M_{B}\left(\left(C_{i}, \mathcal{N}_{i}\right)\right)$ is equivalent to the $T_{B}$ invariance of $i_{C_{i} *} \mathcal{N}_{i}$.

In [DOPWa, Proposition 7.7] we saw that for any curve $C \subset B$ which is finite over $\mathbb{P}^{1}$ and for any line bundle $\mathcal{N}$ on $B$ the image $\boldsymbol{T}_{B}\left(i_{C *} \mathcal{N}\right)$ is 
again a sheaf of the form $i_{\alpha_{B}(C) *}(?)$ for some line bundle ? $\in \operatorname{Pic}\left(\alpha_{B}(C)\right)$. Therefore $\boldsymbol{T}_{B}$ induces a well defined map $\boldsymbol{T}_{C}: \operatorname{Pic}(C) \rightarrow \operatorname{Pic}\left(\alpha_{B}(C)\right)$. Due to this, the $\tau_{B}$-invariance of $\boldsymbol{F} \boldsymbol{M}_{B}((C, \mathcal{N}))$ is equivalent to the following two conditions:

$$
\begin{aligned}
C & =\alpha_{B}(C) \\
\mathcal{N} & =\boldsymbol{T}_{C}(\mathcal{N}) .
\end{aligned}
$$

Lemma 4.1. The linear system $|r e+k f|$ contains smooth $\alpha_{B}$-invariant curves if $r=3, k \geq 3$ or if $r=2$ and $k \geq 2$ is even.

Proof. First of all, from the explicit equations of a spectral curve [FMW97] and Bertini's theorem, it is easy to see that the general curve $C$ in the linear system $|r e+k f|$ will be smooth as long as $k \geq r>1$. The same kind of analysis allows one to understand the $\alpha_{B}$-invariant members of these linear systems as well. Indeed, recall (see e.g. [FMW99]) that for every $a \geq 0$ we have an isomorphism $\beta_{*} \mathcal{O}_{B}(a e)=\mathcal{O}_{\mathbb{P}^{1}} \oplus \mathcal{O}_{\mathbb{P}^{1}}(-2) \oplus \ldots \mathcal{O}_{\mathbb{P}^{1}}(-a)$. In particular, by the projection formula we get isomorphisms

$$
\begin{aligned}
H^{0}\left(B, \mathcal{O}_{B}(e)\right) & =H^{0}\left(\mathbb{P}^{1}, \mathcal{O}_{\mathbb{P}^{1}}\right) \\
H^{0}\left(B, \mathcal{O}_{B}(2 e+2 f)\right) & =H^{0}\left(\mathbb{P}^{1}, \mathcal{O}_{\mathbb{P}^{1}}(2)\right) \oplus H^{0}\left(\mathbb{P}^{1}, \mathcal{O}_{\mathbb{P}^{1}}\right) \\
H^{0}\left(B, \mathcal{O}_{B}(3 e+3 f)\right) & =H^{0}\left(\mathbb{P}^{1}, \mathcal{O}_{\mathbb{P}^{1}}(3)\right) \oplus H^{0}\left(\mathbb{P}^{1}, \mathcal{O}_{\mathbb{P}^{1}}(1)\right) \oplus H^{0}\left(\mathbb{P}^{1}, \mathcal{O}_{\mathbb{P}^{1}}\right) .
\end{aligned}
$$

Let $X \in H^{0}\left(B, \mathcal{O}_{B}(2 e+2 f)\right), Y \in H^{0}\left(B, \mathcal{O}_{B}(3 e+3 f)\right)$ and $Z \in H^{0}\left(B, \mathcal{O}_{B}(e)\right.$ be the preferred sections corresponding to the generator of the piece $H^{0}\left(\mathbb{P}^{1}, \mathcal{O}_{\mathbb{P}^{1}}\right)$ under the above decompositions. Note that in terms of the sections $x \in H^{0}\left(P, \mathcal{O}_{P}(1) \otimes p^{*} \mathcal{O}_{\mathbb{P}^{1}}(2), y \in H^{0}\left(P, \mathcal{O}_{P}(1) \otimes p^{*} \mathcal{O}_{\mathbb{P}^{1}}(3)\right.\right.$ and $z \in H^{0}\left(P, \mathcal{O}_{P}(1)\right.$, which were used in [DOPWa, Section 3.2] to define the Weierstrass model of $B$, we have $x_{\mid W_{\beta}}=X Z, y_{\mid W_{\beta}}=Y, z_{\mid W_{\beta}}=Z^{3}$.

With this notation the isomorphism $H^{0}\left(\mathcal{O}_{\mathbb{P}^{1}}(k)\right) \oplus H^{0}\left(\mathcal{O}_{\mathbb{P}^{1}}(k-2) \oplus \ldots \oplus H^{0}\left(\mathcal{O}_{\mathbb{P}^{1}}(k-r) \rightarrow H^{0}\left(B, \mathcal{O}_{B}(r e+k f)\right)\right.\right.$ is given explicitly by the formula $\left(a_{k}, a_{k-2}, \ldots, a_{k-r}\right) \mapsto\left(\beta^{*} a_{k}\right) Z^{r}+\left(\beta^{*} a_{k-2}\right) X Z^{r-2}+\left(\beta^{*} a_{k-3}\right) Y Z^{r-3}+\ldots$

In particular the curves $C_{2}$ and $C_{3}$ can be identified with the zero loci of

$$
\left(\beta^{*} a_{k_{2}}\right) Z^{2}+\left(\beta^{*} a_{k_{2}-2}\right) X \text { and }\left(\beta^{*} a_{k_{3}}\right) Z^{3}+\left(\beta^{*} a_{k_{3}-2}\right) X Z+\left(\beta^{*} a_{k_{3}-3}\right) Y \text {, }
$$

respectively. 
Now recall, that in [DOPWa, Section 3.2] we identified $\alpha_{B}$ with the involution induced from $\tau_{P \mid W_{\beta}}$ and that $\tau_{P}$ acts trivially on the sections $x$, $y, z$. In view of the comparison formulas $x_{\mid W_{\beta}}=X Z, y_{\mid W_{\beta}}=Y, z_{\mid W_{\beta}}=$ $Z^{3}$, this implies that $\alpha_{B}^{*}(X)=X, \alpha_{B}^{*}(Y)=Y$ and $\alpha_{B}^{*}(Z)=Z$. Here the lifting of the action of $\alpha_{B}$ to an action on line bundles of the form $\mathcal{O}_{B}(r e+k f)$ is chosen in the way described in [DOPWa, Section 3.2]. In particular $\alpha_{B}^{*}\left(\beta^{*} s\right)=\beta^{*}\left(\tau_{\mathbb{P}^{1}}^{*} s\right)$ for any section $s \in \mathcal{O}_{\mathbb{P}^{1}}(k)$.

Since $\alpha_{B}^{*}$ acts linearly on the projective space $|r e+k f|$ it follows that $\alpha_{B}$ will preserve a divisor $C \in|r e+k f|$ if and only if

$$
C \in \mathbb{P}\left(H^{0}\left(B, \mathcal{O}_{B}(r e+k f)\right)^{+}\right) \cup \mathbb{P}\left(H^{0}\left(B, \mathcal{O}_{B}(r e+k f)\right)^{-}\right) \subset|r e+k f|,
$$

where $H^{0}\left(B, \mathcal{O}_{B}(r e+k f)\right)^{ \pm}$denote the \pm 1 eigenspaces of $\alpha_{B}^{*}$ acting on $H^{0}\left(B, \mathcal{O}_{B}(r e+k f)\right)$. Therefore we see that for each $i$ there are two families of $\alpha_{B}$-invariant $C_{i}$ 's, each parameterized by a projective space. In particular we will have $\alpha_{B}\left(C_{i}\right)=C_{i}$ if and only if all the coefficients in the polynomial expressions (4.3) are simultaneously $\tau_{\mathbb{P}^{1}}$-invariant or simulatneously $\tau_{\mathbb{P}^{1}}$-antiinvariant. Now the Bertini theorem immediately implies that we can find a smooth $C_{3}$, which is preserved by $\alpha_{B}$ as long as $k_{3} \geq 3$ and we can find a smooth $C_{2}$, which is preserved by $\alpha_{B}$ as long as $k_{2} \geq 2$ and $k_{2}$ is even.

Remark 4.2. Unfortunately, when $k_{2}$ is odd the linear systems $\left|2 e+k_{2} f\right|^{ \pm}$ will each have a fixed component and so all the $\alpha_{B}$-invariant curves $C_{2}$ will be reducible. In order to see this consider the homogeneous coordinates $\left(t_{0}: t_{1}\right)$ on $\mathbb{P}^{1}$ which were used in [DOPWa, Section 3.2] to define the standard action of $\tau_{\mathbb{P}^{1}}$. In other words $\left(t_{0}: t_{1}\right)$ are such that $\tau_{\mathbb{P}^{1}}^{*}\left(t_{0}\right)=t_{0}, \tau_{\mathbb{P}^{1}}^{*} t_{1}=-t_{1}$ and $0=(1: 0)$ and $\infty=(0: 1)$. Now it is clear that if $a$ is a $\tau_{\mathbb{P}^{1}}$-invariant homogeneous polynomial in $t_{0}$ and $t_{1}$ of odd degree, then $a$ is divisible by

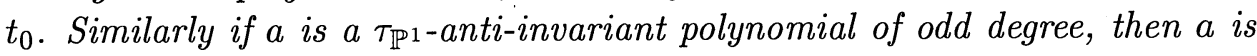
divisible by $t_{1}$. In particular, since $k_{2}$ and $k_{2}-2$ have the same parity we see that for $k_{2}$ odd, the fiber $f_{\infty}$ is the fixed component of the linear system $\left|2 e+k_{2} f\right|^{+}$and the fiber $f_{0}$ is the fixed component of the linear system $\left|2 e+k_{2} f\right|^{-}$.

Lemma 4.3. Let $C$ be an $\alpha_{B}$-invariant curve in $|r e+k f|$ which is finite over $\mathbb{P}^{1}$, and assume that $i_{C}^{*} \operatorname{Pic}(B)$ is dense in $\operatorname{Pic}^{0}(C)$. Then for every $d \in \mathbb{Z}$ there exist line bundles $\mathcal{N} \in \operatorname{Pic}^{d}(C)$, s.t. $\boldsymbol{T}_{C}(\mathcal{N})=\mathcal{N}$. 
Proof. The morphism $\boldsymbol{T}_{C}: \operatorname{Pic}(C) \rightarrow \operatorname{Pic}(C)$ is given explicitly by the formula:

$$
\boldsymbol{T}_{C}(\mathcal{N})=\alpha_{C}^{*}(\mathcal{N}) \otimes \mathcal{O}_{C}\left(e_{9}-e_{1}+f\right)
$$

where $\alpha_{C}=\alpha_{B \mid C}$.

Indeed, by part (b) of [DOPWa, Proposition 7.7] this formula holds for all line bundles $\mathcal{N} \in \operatorname{Pic}\left(i_{C}^{*} \operatorname{Pic}(B)\right)$. By the density assumption it holds for all $\mathcal{N} \in \operatorname{Pic}^{0}(C)$. But applying $\boldsymbol{T}_{C}$ to the short exact sequence

$$
0 \rightarrow \mathcal{N}(-p) \rightarrow \mathcal{N} \rightarrow \mathcal{O}_{p} \rightarrow 0
$$

we find $\boldsymbol{T}_{C}(\mathcal{N}(-p))=\boldsymbol{T}_{C}(\mathcal{N})\left(-\alpha_{C}(p)\right)$, so the formula extends to all components of $\operatorname{Pic}(C)$.

Thus a point $x \in \operatorname{Pic}^{0}(C)$ will be fixed under $\boldsymbol{T}_{C}$ if and only if

$$
x-\alpha_{C}^{*}(x)=\mathcal{O}_{C}\left(e_{9}-e_{1}+f\right) .
$$

This equation is consistent exactly when

$$
\mathcal{O}_{C}\left(e_{9}-e_{1}+f\right) \in \operatorname{im}\left[\operatorname{Pic}^{0}(C) \stackrel{\alpha_{C}^{*}-\mathrm{id}}{\longrightarrow} \operatorname{Pic}^{0}(C)\right] .
$$

Since $\alpha_{C}$ has fixed points on $C$, it follows [Mum74] that $\operatorname{im}\left(\alpha_{C}^{*}-\mathrm{id}\right)=$ $\operatorname{ker}\left(\alpha_{C}^{*}+\mathrm{id}\right)$. But from [DOPWa, Table 1 and Formula (4.2)] we see that

$$
\left(\alpha_{B}^{*}+\mathrm{id}\right)\left(e_{9}-e_{1}+f\right)=2 e_{9}+2 f+e_{7}-\ell=o_{1}+o_{2} .
$$

Since $o_{1}$ and $o_{2}$ do not intersect $C$, this implies that $\mathcal{O}_{C}\left(e_{9}-e_{1}+f\right)$ is $\alpha_{C}^{*}$-anti-invariant. Hence there is a translate of of $\operatorname{Pic}^{0}\left(C / \alpha_{C}\right)$ in $\operatorname{Pic}^{0}(C)$ consisting of solutions of (4.5).

Shifting by an arbitrary multiple of an $\alpha_{C_{i}}$-fixed point, we see that there are $\boldsymbol{T}_{C_{i}}$-fixed points in $\operatorname{Pic}^{d}\left(C_{i}\right)$ for every $d$.

In view of this lemma it only remains to check the density of $i_{C_{i}}^{*} \operatorname{Pic}(B)$ in $\operatorname{Pic}^{0}\left(C_{i}\right)$. We do this only in the cases $\left(i=2, k_{2}=3\right)$ and $\left(i=3, k_{3}=6\right)$, which are the cases needed in section 5 . Unfortunately the statement of Lemma 4.3 does not directly apply to the first of these cases (see Remark 4.2), so we will treat it separately next. 


\subsection{Invariance for $k_{2}=3$}

By Remark 4.2, the general $\alpha_{B}$-invariant curve $C_{2}$ in the linear system $\mid 2 e+$ $3 f \mid$ is of the form $C_{2}=\bar{C}_{2}+F$, where $\bar{C}_{2}$ is a smooth curve in the linear system $|2 e+2 f|^{+}$and $F$ denotes one of the elliptic curves $f_{0}, f_{\infty}$. Assume that $\mathcal{N}_{2} \rightarrow C_{2}$ is a line bundle and let $\overline{\mathcal{N}}_{2}=\mathcal{N}_{2} \otimes \mathcal{O}_{\bar{C}_{2}}$ be its restriction to $\bar{C}_{2}$. We know that $\bar{W}_{2}:=\boldsymbol{F} \boldsymbol{M}_{B}\left(i_{\bar{C}_{2} *} \overline{\mathcal{N}}_{2}\right)$ is a vector bundle. We want $W_{2}:=\boldsymbol{F} \boldsymbol{M}_{B}\left(i_{C_{2} *} \mathcal{N}_{2}\right)$ to be a vector bundle too.

Lemma 4.4. $W_{2}$ is a vector bundle if and only if $\operatorname{deg}\left(\mathcal{N}_{2 \mid F}\right)=1$.

Proof. We have a short exact sequence of torsion sheaves on $B$

$$
0 \rightarrow i_{F *}\left(\mathcal{N}_{2 \mid F}(-D)\right) \rightarrow i_{C_{2} *} \mathcal{N}_{2} \rightarrow i_{\bar{C}_{2} *} \overline{\mathcal{N}}_{2} \rightarrow 0,
$$

where $D \subset F$ is the effective divisor $D=\bar{C}_{2} \cap F$. Let $G:=\mathcal{N}_{2 \mid F}(-D)$. Since $G$ is a line bundle on the fiber $F$ we have $\boldsymbol{F} \boldsymbol{M}_{B}\left(i_{F *} G\right)=i_{F *}\left(\boldsymbol{F} \boldsymbol{M}_{F}(G)\right)$, where $\boldsymbol{F} \boldsymbol{M}_{F}: D^{b}(F) \rightarrow D^{b}(F)$ is the Fourier-Mukai transform with respect to the Poincare bundle $\mathcal{P}_{B \mid F \times F}$. If we apply $\boldsymbol{F} M_{B}$ to the above exact sequence, we will get the long exact sequence of cohomology sheaves

$$
\begin{aligned}
0 \longrightarrow \mathcal{H}^{0}\left(i_{F *} \boldsymbol{F} \boldsymbol{M}_{F}(G)\right) \longrightarrow \mathcal{H}^{0} \boldsymbol{F} \boldsymbol{M}_{B}\left(i_{C_{2} *} \mathcal{N}_{2}\right) \longrightarrow \bar{W}_{2} \\
\hookrightarrow_{\mathcal{H}^{1}\left(i_{F *} \boldsymbol{F} \boldsymbol{M}_{F}(G)\right) \longrightarrow \mathcal{H}^{1} \boldsymbol{F} \boldsymbol{M}_{B}\left(i_{C_{2} *} \mathcal{N}_{2}\right) \longrightarrow 0 .}^{\longrightarrow}
\end{aligned}
$$

Since we want the line bundle $\mathcal{N}_{2} \rightarrow C_{2}$ to be chosen so that $\mathcal{H}^{1} \boldsymbol{F} \boldsymbol{M}_{B}\left(i_{C_{2} *} \mathcal{N}_{2}\right)$ $=0$ and $W_{2}=\mathcal{H}^{0} \boldsymbol{F} \boldsymbol{M}_{B}\left(i_{C_{2} *} \mathcal{N}_{2}\right)$ is a rank two vector bundle on $B$, we must have $\mathcal{H}^{0}\left(i_{F *} \boldsymbol{F} \boldsymbol{M}_{F}(G)\right)=0$ and $\mathcal{H}^{1}\left(i_{F *} \boldsymbol{F} \boldsymbol{M}_{F}(G)\right)$ must be a line bundle on $F$ such that there exists a surjection $\bar{W}_{2 \mid f} \rightarrow \mathcal{H}^{1}\left(i_{F *} \boldsymbol{F} \boldsymbol{M}_{F}(G)\right)$. This can only happen if $G$ has degree -1 on $F$.

We note that in the situation of the lemma $W_{2}$ fits in a short exact sequence

$$
0 \rightarrow W_{2} \rightarrow \bar{W}_{2} \rightarrow i_{F *}\left(G^{\vee}\right) \rightarrow 0,
$$

where $G$ is defined in the proof of the lemma. Indeed, the proof of Lemma 4.4 gave us a short exact sequence

$$
0 \rightarrow W_{2} \rightarrow \bar{W}_{2} \rightarrow i_{F *}\left(\boldsymbol{F} \boldsymbol{M}_{F}(G)[1]\right) \rightarrow 0 .
$$

But every line bundle of degree -1 on an elliptic curve $F$ is of the form $\mathcal{O}_{F}(-p)$ for some point $p \in F$. Applying $\boldsymbol{F} \boldsymbol{M}_{F}$ to the short exact sequence

$$
0 \rightarrow \mathcal{O}_{F}(-p) \rightarrow \mathcal{O}_{F} \rightarrow \mathcal{O}_{p} \rightarrow 0
$$


we see that $\boldsymbol{F} \boldsymbol{M}_{F}(G)=G^{\vee}[-1]$ for any line bundle of degree -1 on $F$.

Given $\left(\bar{C}_{2}, \overline{\mathcal{N}}_{2}\right)$ Lemma 4.4 produces a 2-parameter family of vector bundles $W_{2}$. Indeed, let $G \rightarrow F$ be any line bundle of degree -1 . Consider the semi-stable bundle $\bar{W}_{2 \mid F}$ on $F$. Since generically $\bar{C}_{2}$ intersects $F$ at two distinct points we will have $\bar{W}_{2 \mid F}=A \oplus A^{\vee}$, where $A$ is a non-trivial line bundle of degree zero on $F$. Therefore $h^{0}\left(F, A^{\vee} \otimes G^{\vee}\right)=h^{0}\left(F, A \otimes G^{\vee}\right)=1$ and so we have unique (up to scale) maps $A \rightarrow G^{\vee}$ and $A^{\vee} \rightarrow G^{\vee}$. Also since the degree one line bundles $A^{\vee} \otimes G^{\vee}$ and $A \otimes G^{\vee}$ are not isomorphic, it follows that their unique (up to a scale) sections vanish at different points on $F$. Hence we get a one parameter family of surjective maps of vector bundles $A \oplus A^{\vee} \rightarrow G^{\vee}$. The corresponding Hecke transform of $\bar{W}_{2}$ :

$$
W_{2}=\operatorname{ker}\left[\bar{W}_{2} \rightarrow i_{F *}\left(A \oplus A^{\vee}\right) \rightarrow i_{F *}\left(G^{\vee}\right)\right]
$$

is a rank two vector bundle on $B$ which is the Fourier-Mukai image of a line bundle $\mathcal{N}_{2}$ on $C_{2}=\bar{C}_{2} \cup F$. In particular $W_{2}$ is a deformation of a rank two vector bundle $W$ corresponding to a spectral datum $(C, \mathcal{N})$ where $C$ is a smooth (but non-invariant) curve in the linear system $|2 e+3 f|$ and $\mathcal{N}$ is a line bundle on $C$. Even though this $W$ can not be $\tau_{B}$ invariant, this shows that as far as Chern classes are concerned the bundle $W_{2}$ behaves as a bundle corresponding to a smooth spectral cover.

We are now ready to analyze the $\tau_{B}$-invariance properties of $W_{2}$. First of all, since $\bar{C}_{2}$ is smooth Lemma 4.3 applies modulo the following density statement:

Lemma 4.5. $i_{\bar{C}_{2}}^{*} \operatorname{Pic}(B)$ is Zariski dense in $\operatorname{Pic}^{0}\left(\bar{C}_{2}\right)$.

Proof. The curves $\bar{C}_{2}$ have genus 2 and in the linear system $\mid 2 e+$ $\left.2 f\right|^{+}$we have a degenerate curve consisting of the zero section $e$ taken with multiplicity two and of two fibers of $\beta$ which are exchanged by $\alpha_{B}$. In particular the Jacobian of this degenerate curve is just the product of the two fibers. But the Mordell-Weil group of $B$ has rank 6 and in particular we get elements of infinite order in the general fiber of $\beta$ which are restrictions of global line bundles. By continuity this implies that for a general $\bar{C}_{2} \in \mid 2 e+$ $2 \mathrm{f}^{+}$we can find both $\alpha_{B}$-invariant and $\alpha_{B}$-anti-invariant line bundles on $B$ that restrict to elements of infinite order in $\operatorname{Pic}^{0}\left(\bar{C}_{2}\right)$. Finally $\alpha_{B}$ has two fixed points on a general $\bar{C}_{2}$ and so $g\left(\bar{C}_{2} / \alpha_{\bar{C}_{2}}\right)=1$ and $\operatorname{dim} \operatorname{Prym}\left(\bar{C}_{2}, \alpha_{\bar{C}_{2}}\right)=$ 1. Hence $i_{\bar{C}_{2}}^{*} \operatorname{Pic}(B)$ is dense in $\operatorname{Pic}^{0}\left(\bar{C}_{2}\right)$.

We now reach the main point of this subsection: 
Proposition 4.6. For every integer $d$ there exists a $\tau_{B}$-invariant vector bundle $W_{2}$ whose spectral data $\left(C_{2}, \mathcal{N}_{2} \in \operatorname{Pic}\left(C_{2}\right)\right)$ deforms flatly to a smooth curve in $B$ and a line bundle of degree $d$ on it.

Proof. We have a two parameter family of $\alpha_{B}$-invariant curves $\bar{C}_{2}$. By Lemmas 4.3 and 4.5 , there is a one parameter family of $\boldsymbol{T}_{\bar{C}_{2}}$-invariant line bundles $\overline{\mathcal{N}}_{2}$ on each. Althogether we get a three parameter family of $\tau_{B^{-}}$ invariant bundles $\bar{W}_{2}$. We have seen above that each of these gives rise to a two parameter family of bundles $W_{2}$. We will check now that in each such two parameter family there is a finite number (in fact, four) of $\tau_{B}$ invariant $W_{2}$.

Indeed for every such $\bar{W}_{2}$ we must look for a $\tau_{B}$-invariant Hecke transform $W_{2}$. For this we need to ensure that $G^{\vee}$ is preserved by $\tau_{B \mid F}$ and that the map $\bar{W}_{2} \rightarrow i_{F *}\left(G^{\vee}\right)$ is $\tau_{B}$-equivariant. We have two possibilities: $F=f_{0}$ or $F=f_{\infty}$. Recall that $\tau_{B \mid f_{0}}=t_{\zeta(0)}$ and $\tau_{B \mid f_{\infty}}=t_{\zeta(\infty)} \circ(-1)_{f_{\infty}}$ and that $\zeta(0) \in f_{0}$ and $\zeta(\infty) \in f_{\infty}$ are non-trivial points of order two. In particular $\tau_{B \mid f_{0}}$ does not fix any line bundle of degree one on $f_{0}$ and $\tau_{B \mid f_{\infty}}$ fixes precisely four such bundles, namely the four square roots of the degree two line bundle $\mathcal{O}_{f_{\infty}}(e(\infty)+\zeta(\infty))$.

Choose now $F=f_{\infty}$ and $G^{\vee}$ to be one of the four square roots of $\mathcal{O}_{f_{\infty}}(e(\infty)+\zeta(\infty))$. Choose a non-zero map $s: A \rightarrow G^{\vee}$. Then $\tau_{B \mid f_{\infty}}^{*} s$ : $A^{\vee} \rightarrow G^{\vee}$ is also a non-zero map and so, as before, $s \oplus \tau_{B \mid f_{\infty}}^{*} s: A \oplus A^{\vee} \rightarrow G^{\vee}$ is surjective. Using this map as the center of a Hecke transform, we get a $\tau_{B}$-invariant $W_{2}$.

\subsection{Invariance for $k_{3}=6$}

Let $W_{3}=\boldsymbol{F} \boldsymbol{M}_{B}\left(i_{C_{3} * \mathcal{N}_{3}}\right)$ for some curve in $|3 e+6 f|$. As we saw above, in this case, we can chose $C_{3}$ to be smooth and preserved by $\alpha_{B}$ and so in order to find $\tau_{B}$-invariant $W_{3}$ 's we only need to show that for a general $C_{3} \in|3 e+6 f|^{ \pm}$the image $i_{C_{3}}^{*} \operatorname{Pic}(B)$ will be Zariski dense in $\operatorname{Pic}^{0}\left(C_{3}\right)$.

The Zariski closure of the image $i_{C_{3}}^{*} \operatorname{Pic}(B)$ varies lower-semi-continuously with $C_{3}$, so it suffices to exhibit one good $C_{3}$. Our $C_{3}$ will be reducible, consiting of a generic $\alpha_{B}$-invariant curve $\bar{C}_{2}$ in the linear system $|2 e+2 f|$, plus the zero section $e$, plus two generic fibers $\phi_{1}$ and $\phi_{2}$, plus their images $\phi_{3}:=\alpha_{B}\left(\phi_{1}\right)$ and $\phi_{4}:=\alpha_{B}\left(\phi_{2}\right)$. 
The arithmetic genus of $C_{3}$ is 13 . The 13 -dimensional $\mathrm{Pic}^{0}\left(C_{3}\right)$ is a $\left(\mathbb{C}^{\times}\right)^{7}$ extension of the six dimensional abelian variety $\mathcal{A}:=\operatorname{Pic}^{0}\left(\bar{C}_{2}\right) \times$ $\prod_{i=1}^{4} \mathrm{Pic}^{0}\left(\phi_{i}\right)$. So our density statement follows from the from the following two lemmas.

Lemma 4.7. For a generic choice of $\bar{C}_{2}, \phi_{1}, \phi_{2}$, the image of $i_{C_{3} *} \operatorname{Pic}^{0}(B)$ in $\mathcal{A}$ is Zariski dense.

Lemma 4.8. For a generic choice of $\bar{C}_{2}, \phi_{1}, \phi_{2}$, no proper subgroup of $\operatorname{Pic}^{0}\left(C_{3}\right)$ surjects onto $\mathcal{A}$.

Proof of Lemma 4.7. Under the genericity assumption in the hypothesis of the lemma, it is clear that there are no isogenies among $\phi_{1}, \phi_{2}$, and the two elliptic curves $\operatorname{Pic}^{0}\left(\bar{C}_{2} / \alpha_{\bar{C}_{2}}\right)$ and $\operatorname{Prym}\left(\bar{C}_{2}, \alpha_{\bar{C}_{2}}\right)$. So it suffices to prove density separately in each of the two dimensional abelian varieties $\phi_{1} \times \phi_{3}$, $\phi_{2} \times \phi_{4}$ and $\mathrm{Pic}^{0}\left(\bar{C}_{2}\right)$. Density in $\mathrm{Pic}^{0}\left(\bar{C}_{2}\right)$ was already proved in Lemma 4.5 and density in say $\phi_{1} \times \phi_{3}$ was established during the proof of that result. The lemma is proven.

Proof of Lemma 4.8. Let $\mathcal{B}$ be a $\mathbb{C}^{\times}$-extension of an abelian variety $\mathcal{A}$. Such a $\mathcal{B}$ determines a line bundle $[\mathcal{B}] \in \operatorname{Pic}^{0}(\mathcal{A})$. A proper subgroup of $\mathcal{B}$ surjecting onto $\mathcal{A}$ will exist if and only if $[\mathcal{B}]$ is torsion.

Similarly, our $\left(\mathbb{C}^{\times}\right)^{7}$-extension $\mathrm{Pic}^{0}\left(C_{3}\right)$ will contain a proper subgroup surjecting onto $\mathcal{A}$ if and only there is a non-zero character $\chi:\left(\mathbb{C}^{\times}\right)^{7} \rightarrow \mathbb{C}^{\times}$ such that the associated line bundle $L_{\chi}:=\operatorname{Pic}^{0}\left(C_{3}\right) \times_{\chi} \mathbb{C}$ over $\mathcal{A}$ is torsion.

Therefore it suffices to find seven characters $\chi_{1}, \ldots \chi_{7}$ of $\left(\mathbb{C}^{\times}\right)^{7}$ such that the associated line bundles are linearly independent over $\mathbb{Q}$. For this we will need an intrinsic description of the character lattice $\Lambda$ of $\left(\mathbb{C}^{\times}\right)^{7}$ in terms of the geometry of the curve $C_{3}$. The singular set of $C_{3}$ is $S=\left\{s_{i j} \mid i=\right.$ $1,2,3,4$ and $j=1,2,3\}$, where $\phi_{i} \cap \bar{C}_{2}=\left\{s_{i j}\right\}_{j=1}^{2}$, and $s_{i 3}=\phi_{i} \cap e$. Here the singular points are labelled so that $\alpha_{B}\left(s_{1 j}\right)=s_{3 j}$ and $\alpha_{B}\left(s_{2 j}\right)=s_{4 j}$. Now the lattice $\Lambda$ is explicitly described as:

$$
\Lambda=\operatorname{ker}\left[\mathbb{Z}^{S} \rightarrow \pi_{0}\left(C_{3}-S\right)\right]
$$


Here the map $\mathbb{Z}^{S} \rightarrow \pi_{0}\left(C_{3}-S\right)$ sends the characteristic function $\epsilon_{i j}$ of $s_{i j}$ to the difference $\phi_{i}-\bar{C}_{2}$ for $j=1,2$ and to $\phi_{i}-e$ for $j=3$. Our seven characters $\chi_{k}$ are the following seven elements in $\mathbb{Z}^{S}$ :

$$
\begin{aligned}
& \chi_{1}=\epsilon_{11}+\epsilon_{31}-\epsilon_{12}-\epsilon_{32}, \\
& \chi_{2}=\epsilon_{21}+\epsilon_{41}-\epsilon_{22}-\epsilon_{42}, \\
& \chi_{3}=\epsilon_{11}+\epsilon_{33}-\epsilon_{13}-\epsilon_{31}, \\
& \chi_{4}=\epsilon_{21}+\epsilon_{43}-\epsilon_{23}-\epsilon_{41}, \\
& \chi_{5}=\epsilon_{11}+\epsilon_{32}-\epsilon_{12}-\epsilon_{31}, \\
& \chi_{6}=\epsilon_{21}+\epsilon_{42}-\epsilon_{22}-\epsilon_{41}, \\
& \chi_{7}=\epsilon_{21}+\epsilon_{41}-\epsilon_{11}-\epsilon_{31}+\epsilon_{13}+\epsilon_{33}-\epsilon_{23}-\epsilon_{43} .
\end{aligned}
$$

To prove the independence of the $L_{\chi_{k}}$ over $\mathbb{Q}$ it suffices to prove the independence of the restrictions $\lambda_{k}:=L_{\chi_{k} \mid \prod_{i=1}^{4} \phi_{i}}$. We represent a degree zero line bundle $\lambda$ on $\prod_{i=1}^{4} \phi_{i}$ by a four-tuple $\left(\lambda^{i} \in \operatorname{Pic}^{0}\left(\phi_{i}\right)\right)_{i=1}^{4}$. In this notation the $\lambda_{k}$ 's become:

$$
\begin{aligned}
& \lambda_{1}=(a, 0, a, 0), \\
& \lambda_{2}=(0, b, 0, b), \\
& \lambda_{3}=(c, 0,-c, 0), \\
& \lambda_{4}=(0, d, 0,-d), \\
& \lambda_{5}=(a, 0,-a, 0), \\
& \lambda_{6}=(0, b, 0,-b), \\
& \lambda_{7}=(-c, d,-c, d),
\end{aligned}
$$

where

$$
\begin{aligned}
& a=\mathcal{O}_{\phi_{1}}\left(s_{11}-s_{12}\right) \in \operatorname{Pic}^{0}\left(\phi_{1}\right) \cong \operatorname{Pic}^{0}\left(\phi_{3}\right), \\
& b=\mathcal{O}_{\phi_{2}}\left(s_{21}-s_{22}\right) \in \operatorname{Pic}^{0}\left(\phi_{2}\right) \cong \operatorname{Pic}^{0}\left(\phi_{4}\right), \\
& c=\mathcal{O}_{\phi_{1}}\left(s_{11}-s_{13}\right) \in \operatorname{Pic}^{0}\left(\phi_{1}\right) \cong \operatorname{Pic}^{0}\left(\phi_{3}\right), \\
& d=\mathcal{O}_{\phi_{2}}\left(s_{21}-s_{23}\right) \in \operatorname{Pic}^{0}\left(\phi_{2}\right) \cong \operatorname{Pic}^{0}\left(\phi_{4}\right) .
\end{aligned}
$$

So we only need to show the linear independence over $\mathbb{Q}$ of $a, c \in \operatorname{Pic}^{0}\left(\phi_{1}\right)$ and similarly for $b, d \in \mathrm{Pic}^{0}\left(\phi_{2}\right)$. This however is obvious from the fact that $c, d$ are univalued as functions on the base $\mathbb{P}^{1}$, whereas $a, b$ are two-valued. The lemma is proven.

\section{$5 \quad$ Numerical conditions}

Our goal is to construct a stable rank five holomorphic vector bundle on the Calabi-Yau manifold $Z:=X / \tau_{X}$ which has a trivial determinant, three generations and an anomaly which can be absorbed into $M 5$-branes. In 
terms of $X$ this amounts to finding a rank five vector bundle $V \rightarrow X$ so that:

(S) $V$ is a stable vector bundle.

(I) $V$ is $\tau_{X}$-invariant.

(C1) $c_{1}(V)=0$.

(C2) $c_{2}(X)-c_{2}(V)$ is effective.

(C3) $c_{3}(V)=12$.

We will construct a whole family of $V$ 's satisfying these conditions. As explained in section 3, each $V$ will be constructed as an extension

$$
0 \rightarrow V_{2} \rightarrow V \rightarrow V_{3} \rightarrow 0
$$

where the $V_{i}$ 's have special form. In fact, as we argued in section 3 , in order to satisfy the condition (I) it is sufficient to take

$$
V_{i}=\boldsymbol{F} \boldsymbol{M}_{X}\left(\Sigma_{i},\left(\pi_{\mid \Sigma_{i}}^{\prime}\right)^{*} \mathcal{N}_{i} \otimes \pi^{*} L_{i} \otimes \mathcal{O}_{\Sigma_{i}}\left(-\sum_{k=1}^{i} a_{i k}\left(\left\{p_{i 1 k}\right\} \times n_{1}^{\prime}+\left\{p_{i 2 k}\right\} \times o_{2}^{\prime}\right)\right)\right)
$$

with $a_{i k}$ being positive integers, $\Sigma_{i}=\pi^{*} C_{i}$ for some smooth curve $C_{i} \subset B$ satisfying (4.1) and $\mathcal{N}_{i} \in \operatorname{Pic}^{d_{i}}\left(C_{i}\right)$ satisfying (4.2). In fact, we do not need $C_{i}$ to be smooth; it is sufficient for the pair $\left(C_{i}, \mathcal{N}_{i}\right)$ to be deformable to a pair $\left(C_{i}^{\prime}, \mathcal{N}_{i}^{\prime}\right)$ with $C_{i}^{\prime}$ being smooth but not necessarily $\alpha_{B}$-invariant. Furthermore, we showed in section 3 , that such $\left(C_{i}, \mathcal{N}_{i}\right)$ do exist and move in positive dimensional families, at least for specific values of $k_{i}$. From now on we will assume that $C_{i}$ and $\mathcal{N}_{i}$ are chosen so that they are deformable to a smooth pair and that (4.1) and (4.2) hold.

Next we will rewrite the conditions (S) and (C1-3) as a sequence of numerical constraints on the numbers $k_{i}, d_{i}$ and on the line bundles $L_{i}$. 


\subsection{The Chern classes of $V$}

There are several ways of calculating the Chern classes of the bundles $V_{i}$. One possibility is to use the cohomological Fourier-Mukai transform on $X$. To avoid long and cumbersome calculations of $f m_{X}$ we choose a slightly different approach which utilizes the details of the geometric structure of $V$.

Recall that in section 3 we gave an alternative description of the bundles $V_{i}$ as

$$
V_{i}=\widetilde{W}_{i} \otimes \pi^{*} L_{i}
$$

where $\widetilde{W}_{i}$ is the result of $a_{i k}, k=1, \ldots i$ Hecke transforms of the bundle $\pi^{*} W_{i}$, where $W_{i}=\boldsymbol{F} \boldsymbol{M}_{B}\left(\left(C_{i}, \mathcal{N}_{i}\right)\right)$. Due to Corollary 3.3 and the identity (3.6) we have

$$
\operatorname{ch}\left(\widetilde{W}_{i}\right)=\pi^{*} \operatorname{ch}\left(W_{i}\right)-\left(\sum_{k=1}^{i} a_{i k}\right) \pi^{\prime *}\left(n_{1}^{\prime}+o_{2}^{\prime}\right)-2\left(\sum_{k=1}^{i} a_{i k}^{2}\right)(f \times \mathrm{pt}) .
$$

Next we need the following

Lemma 5.1. Let $C \subset B$ be a smooth curve in the linear system $\mid \mathcal{O}_{B}(r e+$ $m f) \mid$ and let $\mathcal{N} \in \operatorname{Pic}^{d}(C)$. Let $W=\boldsymbol{F} \boldsymbol{M}_{B}((C, \mathcal{N}))$. Then

$$
c h(W)=r+\left(d+\left(\begin{array}{c}
r+1 \\
2
\end{array}\right)-r m-r\right) f-m \cdot \mathrm{pt} .
$$

Proof. The bundle $W$ is defined as the Fourier-Mukai transform of the spectral datum $(C, \mathcal{N})$ on $B$. Explicitly this means that $W=\boldsymbol{F} \boldsymbol{M}_{B}\left(i_{C *} \mathcal{N}\right)$, where $i_{C}: C \hookrightarrow B$ is the inclusion map. In particular

$$
\operatorname{ch}(W)=\boldsymbol{f m}_{B}\left(\operatorname{ch}\left(i_{C *} \mathcal{N}\right)\right)
$$

and so it suffices to calculate $\operatorname{ch}\left(i_{C *} \mathcal{N}\right)$.

By Groethendieck-Riemann-Roch theorem we have

$$
\begin{aligned}
\operatorname{ch}\left(i_{C *} \mathcal{N}\right) & =\left(i_{C *}(\operatorname{ch}(\mathcal{N}) t d(C))\right) t d(B)^{-1} \\
& =\left(i_{C *}(1+(d+1-g) \cdot \mathrm{pt})\right) \cdot\left(1+\frac{1}{2} f+\mathrm{pt}\right)^{-1} \\
& =((r e+m f)+(d+1-g) \cdot \mathrm{pt})) \cdot\left(1-\frac{1}{2} f-\mathrm{pt}\right) \\
& =(r e+m f)+\left(d+1-g-\frac{r}{2}\right) \cdot \mathrm{pt} .
\end{aligned}
$$


Also by adjunction we have $2 g-2=C \cdot\left(K_{B}+C\right)=2 r m-r^{2}-r$. Hence

$$
\operatorname{ch}\left(i_{C *} \mathcal{N}\right)=(r e+m f)+\left(d+\left(\begin{array}{c}
r+1 \\
2
\end{array}\right)-r m-\frac{r}{2}\right) \cdot \mathrm{pt} .
$$

Finally using [DOPWa, Table 2] we get

$$
\begin{aligned}
\operatorname{ch}(W) & =\boldsymbol{f} \boldsymbol{m}_{B}\left(\operatorname{ch}\left(i_{C *} \mathcal{N}\right)\right) \\
& =\boldsymbol{f} \boldsymbol{m}_{B}\left((r e+m f)+\left(d+\left(\begin{array}{c}
r+1 \\
2
\end{array}\right)-r m-\frac{r}{2}\right) \cdot \mathrm{pt}\right) \\
& =r\left(1-\frac{1}{2} f\right)+m \cdot(-\mathrm{pt})+\left(d+\left(\begin{array}{c}
r+1 \\
2
\end{array}\right)-r m-\frac{r}{2}\right) \cdot f \\
& =r+\left(d+\left(\begin{array}{c}
r+1 \\
2
\end{array}\right)-r m-r\right) f-m \cdot \mathrm{pt} .
\end{aligned}
$$

The lemma is proven.

Remark 5.2. Since the Chern classes are topological invariants, the conclusion of the previous lemma still holds even if $C$ is singular, as long as the sheaf $i_{C *} \mathcal{N}$ deforms flatly to a line bundle on some smooth spectral curve in the same linear system. In particular it will hold for the $W_{2}$ from section 4.2.

Going back to the calculation of $\operatorname{ch}(V)$ let us write $S_{i}^{\alpha}$ for the Newton sums

$$
S_{i}^{\alpha}=\sum_{k=1}^{i} a_{i k}^{\alpha} .
$$

In this notation we need to calculate the product

$$
\operatorname{ch}\left(V_{i}\right)=\left(\pi^{*} \operatorname{ch}\left(W_{i}\right)-S_{i}^{1} \pi^{*}\left(n_{1}^{\prime}+o_{2}^{\prime}\right)-2 S_{i}^{2}(f \times \mathrm{pt})\right) \cdot \operatorname{ch}\left(\pi^{*} L_{i}\right) .
$$

But

$$
\operatorname{ch}\left(\pi^{*} L_{i}\right)=\pi^{*} \operatorname{ch}\left(L_{i}\right)=1+\pi^{*} L_{i}+\frac{L_{i}^{2}}{2}(f \times \mathrm{pt})
$$

and so

- $\pi^{*}\left(n_{1}^{\prime}+o_{2}^{\prime}\right) \cdot \operatorname{ch}\left(\pi^{*} L_{i}\right)=\pi^{*}\left(n_{1}^{\prime}+o_{2}^{\prime}\right)+\left(L_{i} \cdot\left(n_{1}^{\prime}+o_{2}^{\prime}\right)\right) \cdot(f \times \mathrm{pt})$;

- $(f \times \mathrm{pt}) \cdot \operatorname{ch}\left(\pi^{*} L_{i}\right)=f \times \mathrm{pt}$; 
- Lemma 5.1 yields

$$
\begin{aligned}
& \pi^{\prime *} \operatorname{ch}\left(W_{i}\right) \cdot \operatorname{ch}\left(\pi^{*} L_{i}\right)= \\
&=\pi^{\prime *}\left(i+\left(d_{i}+\left(\begin{array}{c}
i+1 \\
2
\end{array}\right)-k_{i} i-i\right) \cdot f-k_{i} \cdot \mathrm{pt}\right) \cdot\left(1+\pi^{*} L_{i}+\frac{L_{i}^{2}}{2}(f \times \mathrm{pt})\right) \\
&=i+\pi^{*}\left(i L_{i}+\left(d_{i}+\left(\begin{array}{c}
i+1 \\
2
\end{array}\right)-k_{i} i-i\right) f^{\prime}\right)+ \\
& \quad+\left[\left(\frac{i}{2} L_{i}^{2}+\left(d_{i}+\left(\begin{array}{c}
i+1 \\
2
\end{array}\right)-k_{i} i-i\right) \cdot\left(L_{i} \cdot f^{\prime}\right)\right)(f \times \mathrm{pt})-k_{i}\left(\mathrm{pt} \times f^{\prime}\right)\right] \\
&-k_{i}\left(L_{i} \cdot f^{\prime}\right) \mathrm{pt} .
\end{aligned}
$$

Combining these formulas we get formulas for $\operatorname{ch}\left(V_{2}\right)$ and $\operatorname{ch}\left(V_{3}\right)$ :

$$
\begin{aligned}
\operatorname{ch}\left(V_{2}\right) & =2+\pi^{*}\left(2 L_{2}+\left(d_{2}-2 k_{2}+1\right) f^{\prime}-S_{2}^{1}\left(n_{1}^{\prime}+o_{2}^{\prime}\right)\right) \\
& +\left(L_{2}^{2}+\left(d_{2}-2 k_{2}+1\right)\left(L_{2} \cdot f^{\prime}\right)-S_{2}^{1}\left(L_{2} \cdot\left(n_{1}^{\prime}+o_{2}^{\prime}\right)\right)-2 S_{2}^{2}\right) \cdot(f \times \mathrm{pt}) \\
& \quad-k_{2}\left(\mathrm{pt} \times f^{\prime}\right) \\
& -k_{2}\left(L_{2} \cdot f^{\prime}\right) \mathrm{pt} .
\end{aligned}
$$

and similarly

$$
\begin{aligned}
\operatorname{ch}\left(V_{3}\right) & =3+\pi^{*}\left(3 L_{3}+\left(d_{3}-3 k_{2}+3\right) f^{\prime}-S_{3}^{1}\left(n_{1}^{\prime}+o_{2}^{\prime}\right)\right) \\
& +\left(\frac{3}{2} L_{3}^{2}+\left(d_{3}-3 k_{3}+3\right)\left(L_{3} \cdot f^{\prime}\right)-S_{3}^{1}\left(L_{3} \cdot\left(n_{1}^{\prime}+o_{2}^{\prime}\right)\right)-2 S_{3}^{2}\right) \cdot(f \times \mathrm{pt}) \\
& \quad-k_{3}\left(\mathrm{pt} \times f^{\prime}\right) \\
& -k_{3}\left(L_{3} \cdot f^{\prime}\right) \mathrm{pt} .
\end{aligned}
$$

Together these formulas give

$$
\begin{aligned}
\operatorname{ch}(V) & =5+ \\
& +\pi^{*}\left(2 L_{2}+3 L_{3}+\left(d_{2}+d_{3}-2 k_{2}-3 k_{3}+4\right) f^{\prime}-\left(S_{2}^{1}+S_{3}^{1}\right)\left(n_{1}^{\prime}+o_{2}^{\prime}\right)\right) \\
& +\left[\left(L_{2}^{2}+(3 / 2) L_{3}^{2}+\left(d_{2}-2 k_{2}+1\right)\left(L_{2} \cdot f^{\prime}\right)+\left(d_{3}-3 k_{3}+3\right)\left(L_{3} \cdot f^{\prime}\right)\right.\right. \\
& \left.-\left(S_{2}^{1} L_{2}+S_{3}^{1} L_{3}\right) \cdot\left(n_{1}^{\prime}+o_{2}^{\prime}\right)-2\left(S_{2}^{2}+S_{3}^{2}\right)\right)(f \times \mathrm{pt}) \\
& \left.\quad-\left(k_{2}+k_{3}\right)\left(\mathrm{pt} \times f^{\prime}\right)\right] \\
& -\left(\left(k_{2} L_{2}+k_{3} L_{3}\right) \cdot f^{\prime}\right) \mathrm{pt} .
\end{aligned}
$$

Therefore, taking into account that $c_{2}(X)=12\left(f \times \mathrm{pt}+\mathrm{pt} \times f^{\prime}\right)$ we see that the conditions (C1-3) translate into the following numerical constraints:

$(\# \mathbf{C 1}) 2 L_{2}+3 L_{3}=\left(S_{2}^{1}+S_{3}^{1}\right)\left(n_{1}^{\prime}+o_{2}^{\prime}\right)-\left(d_{2}+d_{3}-2 k_{2}-3 k_{3}+4\right) f^{\prime}$. 
$(\# \mathbf{C 2 f}) k_{2}+k_{3} \leq 12$

$\left(\# \mathbf{C 2} f^{\prime}\right) L_{2}^{2}+(3 / 2) L_{3}^{2}+\left(d_{2}-2 k_{2}+1\right)\left(L_{2} \cdot f^{\prime}\right)+\left(d_{3}-3 k_{3}+3\right)\left(L_{3} \cdot f^{\prime}\right)-$ $\left(S_{2}^{1} L_{2}+S_{3}^{1} L_{3}\right)\left(n_{1}^{\prime}+o_{2}^{\prime}\right)-2\left(S_{2}^{2}+S_{3}^{2}\right) \geq-12$.

(\#C3) $k_{2}\left(L_{2} \cdot f^{\prime}\right)+k_{3}\left(L_{3} \cdot f^{\prime}\right)=-6$.

Our next task is to express the stability of $V$ in a numerical form.

\subsection{Stability of $V$}

We need to make sure that the bundle $V$ is Mumford stable with respect to some ample class $H \in H^{2}(X, \mathbb{Z})$. Recall (see e.g. [FMW99, Section 7]) that a polarization $H \in H^{2}(X, \mathbb{Z})$ is called suitable if up to an overall scale the components of the fibers of $\pi: X \rightarrow B^{\prime}$ have sufficiently small volume. Starting with any polarization $H_{0} \in H^{2}(X, \mathbb{Z})$ we can construct a suitable polarization by fixing some polarization $h^{\prime} \in H^{2}\left(B^{\prime}, \mathbb{Z}\right)$ and taking

$$
H:=H_{0}+n \cdot \pi^{*} h^{\prime},
$$

with $n \gg 0$. As explained in [FMW99, Theorem 7.1] for a suitable $H$ every vector bundle on $X$ which comes from a spectral cover will be $H$-stable on $X$. From now on we will always assume that $H=H_{0}+n \cdot \pi^{*} h^{\prime}$ is chosen to be suitable. For a torsion free sheaf $\mathcal{F}$ on $X$ denote by $\mu_{H}(\mathcal{F})$ the $H$-slope of $\mathcal{F}$, i.e. $\mu_{H}(\mathcal{F})=\left(c_{1}(\mathcal{F}) \cdot H^{2}\right) / \operatorname{rk}(\mathcal{F})$. By repeating the argument in the proof of [FMW99, Theorem 7.1] one gets the following lemma whose proof is left to the reader.

Lemma 5.3. The bundle $V$ constructed in the previous section is $H$-stable if and only if

(i) The extension

$$
0 \rightarrow V_{2} \rightarrow V \rightarrow V_{3} \rightarrow 0,
$$

is non-split.

(ii) $\mu_{H}\left(V_{2}\right)<\mu_{H}(V)=0$.

Next we express both these conditions into a numerical form. Note that an extension

$$
0 \rightarrow V_{2} \rightarrow V \rightarrow V_{3} \rightarrow 0
$$


will be non-split if and only if it corresponds to a non-zero element in $\operatorname{Ext}^{1}\left(V_{3}, V_{2}\right)=H^{1}\left(X, V_{3}^{\vee} \otimes V_{2}\right)$. Thus we only need to ensure that $H^{1}\left(X, V_{3}^{\vee} \otimes\right.$ $\left.V_{2}\right) \neq 0$. We have the following

Lemma 5.4. For $V_{2}$ and $V_{3}$ as above one has $H^{1}\left(X, V_{3}^{\vee} \otimes V_{2}\right) \neq 0$ if $L_{2} \cdot f^{\prime}>$ $L_{3} \cdot f^{\prime}$.

Proof. We are assuming that the $W_{i}$ 's deform to vector bundles on $B$ coming from smooth spectral covers. So by the upper-semi-continuity of $H^{1}\left(X, V_{2}^{\vee} \otimes V_{3}\right)$ it is enough to prove the lemma for $W_{i}$ 's arising from smooth spectral covers.

Let $L=L_{3}^{-1} \otimes L_{2}$. Then

$$
V_{3}^{\vee} \otimes V_{2}=\widetilde{W}_{3}^{\vee} \otimes \widetilde{W}_{2} \otimes \pi^{*} L
$$

To calculate $H^{1}\left(X, \widetilde{W}_{3}^{\vee} \otimes \widetilde{W}_{2} \otimes \pi^{*} L\right)$ we use the Leray spectral sequence for the projection $\pi: X \rightarrow B^{\prime}$. It yields an exact sequence of vector spaces

$$
\begin{gathered}
0 \longrightarrow H^{1}\left(B^{\prime}, \pi_{*}\left(\widetilde{W}_{3}^{\vee} \otimes \widetilde{W}_{2}\right) \otimes L\right) \longrightarrow H^{1}\left(X, V_{3}^{\vee} \otimes V_{2}\right) \\
H^{0}\left(B^{\prime}, R^{1} \pi_{*}\left(\widetilde{W}_{3}^{\vee} \otimes \widetilde{W}_{2}\right) \otimes L\right) \longrightarrow H^{2}\left(B^{\prime}, \pi_{*}\left(\widetilde{W}_{3}^{\vee} \otimes \widetilde{W}_{2}\right) \otimes L\right) .
\end{gathered}
$$

By construction $\widetilde{W}_{2}$ and $\widetilde{W}_{3}$ are vector bundles on $X$ coming from the spectral construction applied to line bundles on smooth spectral covers, which are also finite over $B^{\prime}$. In particular the restriction of $\widetilde{W}_{3}^{\mathrm{V}} \otimes \widetilde{W}_{2}$ to the general fiber of $\pi$ is regular and semistable of degree zero. Hence for general $\widetilde{W}_{2}$ and $\widetilde{W}_{3}$ we have

- $\pi_{*}\left(\widetilde{W}_{3}^{\vee} \otimes \widetilde{W}_{2}\right)=0$

- $R^{1} \pi_{*}\left(\widetilde{W}_{3}^{\vee} \otimes \widetilde{W}_{2}\right)$ is supported on a curve in $B^{\prime}$ and is a line bundle on its support.

Therefore

$$
H^{1}\left(X, V_{3}^{\vee} \otimes V_{2}\right)=H^{0}\left(B^{\prime}, R^{1} \pi_{*}\left(\widetilde{W}_{3}^{\vee} \otimes \widetilde{W}_{2}\right) \otimes L\right) .
$$

To calculate the latter space notice that $R^{1} \pi_{*}\left(\widetilde{W}_{3}^{\vee} \otimes \widetilde{W}_{2}\right)$ is supported on the curve

$$
\pi\left((-1)_{X}^{*} \Sigma_{3} \cap \Sigma_{2}\right) \subset B^{\prime}
$$

Since $\Sigma_{i}=\pi{ }^{*} C_{i}$, this implies

$$
(-1)_{X}^{*} \Sigma_{3} \cap \Sigma_{2}=\coprod_{t \in(-1)_{B}^{*} C_{3} \cap C_{2}}\{t\} \times f^{\prime} .
$$


Without a loss of generality we may assume that all $t \in(-1)_{B}^{*} C_{3} \cap C_{2}$ project to distinct points in $\mathbb{P}^{1}$ under the map $\beta: B \rightarrow \mathbb{P}^{1}$. Consequently

$$
R^{1} \pi_{*}\left(\widetilde{W}_{3}^{\vee} \otimes \widetilde{W}_{2}\right)=\oplus_{t \in(-1)_{B}^{*} C_{3} \cap C_{2}} \Phi_{t},
$$

where $\Phi_{t}$ is a line bundle on the curve $\pi\left(\{t\} \times f^{\prime}\right)=f_{\beta(t)}^{\prime}$. The line bundle $\Phi_{t}$ depends only on the the restriction of $\widetilde{W}_{3}^{\vee} \otimes \widetilde{W}_{2}$ to the surface $f_{\beta(t)} \times f_{\beta(t)}^{\prime}$. Let $\mathrm{pr}_{1}: f_{\beta(t)} \times f_{\beta(t)}^{\prime} \rightarrow f_{\beta(t)}$ and $\mathrm{pr}_{2}: f_{\beta(t)} \times f_{\beta(t)}^{\prime} \rightarrow f_{\beta(t)}^{\prime}$ denote the natural projections. Then we have

$$
\begin{aligned}
\Phi_{t} & =R^{1} \operatorname{pr}_{2 *}\left(\left(\widetilde{W}_{3}^{\vee} \otimes \widetilde{W}_{2}\right)_{\mid f_{\beta(t)} \times f_{\beta(t)}^{\prime}}\right)=R^{1} \operatorname{pr}_{2 *}\left(\operatorname{pr}_{1}^{*}\left(W_{3}^{\vee} \otimes W_{2}\right)\right) \\
& =R^{1} \operatorname{pr}_{2 *}\left(\operatorname{pr}_{1}^{*} \mathcal{O}_{f_{\beta(t)}}\right)=H^{1}\left(f_{\beta(t)}, \mathcal{O}_{f_{\beta(t)}}\right) \otimes \mathcal{O}_{f_{\beta(t)}^{\prime}} .
\end{aligned}
$$

In other words $H^{1}\left(X, V_{3}^{\vee} \otimes V_{2}\right) \neq 0$ if and only if $L_{\mid f_{\beta(t)}^{\prime}}$ is effective for some $t \in(-1)_{B}^{*} C_{3} \cap C_{2}$. For this it suffices to have $L \cdot f^{\prime}>0$, and it is necessary to have $L \cdot f^{\prime} \geq 0$.

However we saw in the previous section that the condition (C1) implies $2 L_{2} \cdot f^{\prime}+3 L_{3} \cdot f^{\prime}=0$. If we assume that $L \cdot f^{\prime}=0$, then we will get $L_{2} \cdot f^{\prime}=L_{3} \cdot f^{\prime}=0$ which contradicts (C3). Thus $H^{1}\left(X, V_{3}^{\vee} \otimes V_{2}\right) \neq 0$ iff $L \cdot f^{\prime}>0$. The lemma is proven.

Expressing the slope condition in numerical terms is completely straightforward. In the previous section we showed that

$$
c_{1}\left(V_{2}\right)=\pi^{*}\left(2 L_{2}+\left(d_{2}+1-2 k_{2}\right) f^{\prime}-S_{2}^{1}\left(n_{1}^{\prime}+o_{2}^{\prime}\right)\right) .
$$

Hence $\mu_{H}\left(V_{2}\right)<0$ if and only if

$$
\pi^{*}\left(2 L_{2}+\left(d_{2}+1-2 k_{2}\right) f^{\prime}-S_{2}^{1}\left(n_{1}^{\prime}+o_{2}^{\prime}\right)\right) \cdot H^{2}<0 .
$$

It is more convenient to rewrite this as a condition on the surface $B^{\prime}$. Recall that we take $H$ to be of the form

$$
H=H_{0}+n \cdot \pi^{*} h^{\prime}
$$

where $h^{\prime} \in H^{2}\left(B^{\prime}, \mathbb{Z}\right)$ is some polarization and $n \gg 0$. Since $X=B \times_{\mathbb{P}^{1}} B^{\prime}$ we see that any polarization $H_{0}$ on $X$ can be written as

$$
H_{0}=\pi^{*} h_{0}+\pi^{*} h_{0}^{\prime}
$$

for some polarizations $h_{0} \in H^{2}(B, \mathbb{Z})$ and $h_{0}^{\prime} \in H^{2}\left(B^{\prime}, \mathbb{Z}\right)$. In particular

$$
\begin{aligned}
H^{2} & =\left(\pi^{*} h_{0}+\pi^{*} h_{0}^{\prime}\right)^{2}+2 n\left(\pi^{*} h_{0}+\pi^{*} h_{0}^{\prime}\right) \pi^{*} h^{\prime}+n^{2} \cdot \pi^{*}\left(h^{\prime 2}\right) \\
& =\left(h_{0}^{2}\right) \cdot\left(\{\mathrm{pt}\} \times f^{\prime}\right)+2\left(\pi^{*} h_{0}^{\prime}+n \cdot \pi^{*} h^{\prime}\right) \pi^{\prime *} h_{0}+\pi^{*}\left(\left(k_{0}^{\prime}+n \cdot h^{\prime}\right)^{2}\right) .
\end{aligned}
$$


By the projection formula we get

$$
\begin{aligned}
\mu_{H}\left(V_{2}\right)= & \pi^{*}\left(2 L_{2}+\left(d_{2}+1-2 k_{2}\right) f^{\prime}-S_{2}^{1}\left(n_{1}^{\prime}+o_{2}^{\prime}\right)\right) \cdot H^{2} \\
= & \left(2 L_{2}+\left(d_{2}+1-2 k_{2}\right) f^{\prime}-S_{2}^{1}\left(n_{1}^{\prime}+o_{2}^{\prime}\right)\right) \cdot \pi_{*}\left(H^{2}\right) \\
= & \left(2 L_{2}+\left(d_{2}+1-2 k_{2}\right) f^{\prime}-S_{2}^{1}\left(n_{1}^{\prime}+o_{2}^{\prime}\right)\right) \\
& \quad \cdot\left(\left(h_{0}^{2}\right) f^{\prime}+2\left(h_{0}^{\prime}+n \cdot h^{\prime}\right) \pi_{*} \pi^{\prime *} h_{0}\right) \\
= & \left(2 L_{2}+\left(d_{2}+1-2 k_{2}\right) f^{\prime}-S_{2}^{1}\left(n_{1}^{\prime}+o_{2}^{\prime}\right)\right) \cdot\left(\left(h_{0}^{2}\right) f^{\prime}+2\left(h_{0} \cdot f\right) h_{0}^{\prime}\right) \\
& \quad+2\left(h_{0} \cdot f\right) n\left(2 L_{2}+\left(d_{2}+1-2 k_{2}\right) f^{\prime}-S_{2}^{1}\left(n_{1}^{\prime}+o_{2}^{\prime}\right)\right) \cdot h^{\prime} .
\end{aligned}
$$

To derive the last identity we used (3.1) to write

$$
\pi_{*} \pi^{*} h_{0}=\beta^{*} \beta_{*} h_{0}=\left(h_{0} \cdot f\right) \cdot 1+m f^{\prime} \in H^{\bullet}\left(B^{\prime}, \mathbb{Z}\right),
$$

with $m$ being a positive integer. This implies that $\alpha \cdot \pi_{*} \pi^{*} h_{0}=\left(h_{0} \cdot f\right)(\alpha$. 1) $+m\left(\alpha \cdot f^{\prime}\right)$ for any cohomology class $\alpha$ on $B^{\prime}$. In particular pt $\cdot \pi_{*} \pi^{*} h_{0}=$ $\left(h_{0} \cdot f\right) \mathrm{pt}$ and hence the above formula.

In conclusion, we see that for $n \gg 0$ we have

$$
\mu_{H}\left(V_{2}\right)<2\left(h_{0} \cdot f\right) n\left(2 L_{2}+\left(d_{2}+1-2 k_{2}\right) f^{\prime}-S_{2}^{1}\left(n_{1}^{\prime}+o_{2}^{\prime}\right)\right) \cdot h^{\prime},
$$

and so $\mu_{H}\left(V_{2}\right)<0$ provided that

$$
\left(2 L_{2}+\left(d_{2}+1-2 k_{2}\right) f^{\prime}-S_{2}^{1}\left(n_{1}^{\prime}+o_{2}^{\prime}\right)\right) \cdot h^{\prime}<0 .
$$

We are now ready to list all the conditions on $V$ in a numerical form.

\subsection{The list of constraints}

In the previous two sections we translated the conditions (S), (I), (C1-3) into a set of numerical conditions. Together those read:

$(\# \mathbf{S} e) L_{2} \cdot f^{\prime}>L_{3} \cdot f^{\prime}$.

$(\# \mathbf{S} s)\left(2 L_{2}+\left(d_{2}+1-2 k_{2}\right) f^{\prime}-S_{2}^{1}\left(n_{1}^{\prime}+o_{2}^{\prime}\right)\right) \cdot h^{\prime}<0$ for some ample class $h^{\prime} \in \operatorname{Pic}\left(B^{\prime}\right)$.

(\#I) $\tau_{B}^{*} L_{i}=L_{i}$, for $i=2,3$.

$(\# \mathbf{C 1}) 2 L_{2}+3 L_{3}=\left(S_{2}^{1}+S_{3}^{1}\right)\left(n_{1}^{\prime}+o_{2}^{\prime}\right)-\left(d_{2}+d_{3}-2 k_{2}-3 k_{3}+4\right) f^{\prime}$.

$(\# \mathbf{C 2} f) k_{2}+k_{3} \leq 12$. 
$\left(\# \mathbf{C 2} f^{\prime}\right) L_{2}^{2}+(3 / 2) L_{3}^{2}+\left(d_{2}-2 k_{2}+1\right)\left(L_{2} \cdot f^{\prime}\right)+\left(d_{3}-3 k_{3}+3\right)\left(L_{3} \cdot f^{\prime}\right)-$ $\left(S_{2}^{1} L_{2}+S_{3}^{1} L_{3}\right)\left(n_{1}^{\prime}+o_{2}^{\prime}\right)-2\left(S_{2}^{2}+S_{3}^{2}\right) \geq-12$.

(\#C3) $k_{2}\left(L_{2} \cdot f^{\prime}\right)+k_{3}\left(L_{3} \cdot f^{\prime}\right)=-6$.

Observe that these conditions already constrain severely the possible values of $k_{2}, k_{3}, L_{2} \cdot f^{\prime}$ and $L_{3} \cdot f^{\prime}$. Indeed, intersecting both sides of (\#C1) with the curve $f^{\prime} \subset B^{\prime}$ we see that $2\left(L_{2} \cdot f^{\prime}\right)+3\left(L_{3} \cdot f^{\prime}\right)=0$. Recall also that we showed in section 4 that for the existence of smooth curves $C_{2}$ and $C_{3}$ one needs to take $k_{2} \geq 2$ and $k_{3} \geq 3$. Thus the integers $k_{2}, k_{3}, L_{2} \cdot f^{\prime}$ and $L_{3} \cdot f^{\prime}$ should satisfy:

- $L_{2} \cdot f^{\prime}>L_{3} \cdot f^{\prime}$

- $k_{2} \geq 2$ and $k_{3} \geq 3$

- $k_{2}+k_{3} \leq 12$;

- $2\left(L_{2} \cdot f^{\prime}\right)+3\left(L_{3} \cdot f^{\prime}\right)=0$;

- $k_{2}\left(L_{2} \cdot f^{\prime}\right)+k_{3}\left(L_{3} \cdot f^{\prime}\right)=-6$.

Solving these, we find the following finite list of values for $k_{2}, k_{3}, L_{2} \cdot f^{\prime}$ and $L_{3} \cdot f^{\prime}$.

\begin{tabular}{|c|c||c|c|}
\hline$k_{2}$ & $k_{3}$ & $L_{2} \cdot f^{\prime}$ & $L_{3} \cdot f^{\prime}$ \\
\hline \hline 2 & 4 & 9 & -6 \\
\hline 2 & 6 & 3 & -2 \\
\hline 3 & 5 & 18 & -12 \\
\hline 3 & 6 & 6 & -4 \\
\hline 4 & 7 & 9 & -6 \\
\hline
\end{tabular}

Table 1: Possible values of $k_{2}, k_{3}, L_{2} \cdot f^{\prime}, L_{3} \cdot f^{\prime}$

\subsection{Some solutions}

In this section we show that the numerical constraints (\#) can all be satisfied. In fact, we find infinitely many solutions of (\#). These represent an infinite sequence of of moduli spaces (of arbitrarily large dimension) of all possible $V$ 's. 
Fix $k_{2}$ and $k_{3}$ from the values in Table 1 . For such a choice the corresponding numbers $L_{2} \cdot f^{\prime}$ and $L_{3} \cdot f^{\prime}$ are the solutions to the linear system

$$
\left(\begin{array}{cc}
2 & 3 \\
k_{2} & k_{3}
\end{array}\right) \cdot\left(\begin{array}{l}
L_{2} \cdot f^{\prime} \\
L_{3} \cdot f^{\prime}
\end{array}\right)=\left(\begin{array}{c}
0 \\
-6
\end{array}\right)
$$

Thus in terms of $k_{2}$ and $k_{3}$ we have

$$
L_{2} \cdot f^{\prime}=\frac{18}{2 k_{3}-3 k_{2}}, \quad L_{3} \cdot f^{\prime}=\frac{-12}{2 k_{3}-3 k_{2}} .
$$

Put $k=2 k_{3}-3 k_{2}$. Express $L_{i}, i=2,3$ in terms of the standard classes on $B^{\prime}$ as follows

$$
\begin{aligned}
& L_{2}=+\frac{9}{k}\left(e^{\prime}+\zeta^{\prime}\right)+x_{2} f^{\prime}+y_{2}\left(n_{1}^{\prime}+o_{2}^{\prime}\right)+3 M \\
& L_{3}=-\frac{6}{k}\left(e^{\prime}+\zeta^{\prime}\right)+x_{3} f^{\prime}+y_{3}\left(n_{1}^{\prime}+o_{2}^{\prime}\right)-2 M .
\end{aligned}
$$

The most general way to make the $L_{i}$ 's satisfy (\#I) together with (5.2) is to take $M$ to be $\tau_{B}$-invariant and perpendicular to $e^{\prime}+\zeta^{\prime}, f^{\prime}$ and $n_{1}^{\prime}+o_{2}^{\prime}$. This follows from the fact that the intersection form on $H^{2}\left(B^{\prime}, \mathbb{Z}\right)$ is nondegenerate on the span of $e^{\prime}+\zeta^{\prime}, f^{\prime}$ and $n_{1}^{\prime}+o_{2}^{\prime}$. This is evident from Table 2

\begin{tabular}{|c||c|c|c|}
\hline & $e^{\prime}+\zeta^{\prime}$ & $f^{\prime}$ & $n_{1}^{\prime}+o_{2}^{\prime}$ \\
\hline \hline$e^{\prime}+\zeta^{\prime}$ & -2 & 2 & 2 \\
\hline$f^{\prime}$ & 2 & 0 & 0 \\
\hline$n_{1}^{\prime}+o_{2}^{\prime}$ & 2 & 0 & -4 \\
\hline
\end{tabular}

Table 2: Intersection pairing on $e^{\prime}+\zeta^{\prime}, f^{\prime}$ and $n_{1}^{\prime}+o_{2}^{\prime}$

The condition (\#C1) translates into

$$
\begin{aligned}
& 2 x_{2}+3 x_{3}=-\left(d_{2}+d_{3}-2 k_{2}-3 k_{3}+4\right) \\
& 2 y_{2}+3 y_{3}=S_{2}^{1}+S_{3}^{1} .
\end{aligned}
$$

Using Table 2 we compute

$$
\begin{aligned}
L_{2}^{2} & =-\frac{162}{k^{2}}-4 y_{2}^{2}+9 M^{2}+\frac{36}{k}\left(x_{2}+y_{2}\right) \\
L_{3}^{2} & =-\frac{72}{k^{2}}-4 y_{3}^{2}+4 M^{2}-\frac{24}{k}\left(x_{3}+y_{3}\right) \\
\left(n_{1}^{\prime}+o_{2}^{\prime}\right) \cdot L_{2} & =+\frac{18}{k}-4 y_{2} \\
\left(n_{1}^{\prime}+o_{2}^{\prime}\right) \cdot L_{3} & =-\frac{12}{k}-4 y_{3} .
\end{aligned}
$$


So the condition $\left(\# \mathbf{C 2} f^{\prime}\right)$ becomes

$$
\begin{aligned}
-\frac{270}{k^{2}}- & 4 y_{2}^{2}-6 y_{3}^{2}+15 M^{2}+\frac{36}{k}\left(x_{2}+y_{2}-x_{3}-y_{3}\right) \\
& +\frac{1}{k}\left(18 d_{2}-12 d_{3}-36 k_{2}+36 k_{3}-18\right) \\
& -S_{2}^{1}\left(\frac{18}{k}-4 y_{2}\right)-S_{3}^{1}\left(-\frac{12}{k}-4 y_{3}\right)-2\left(S_{2}^{2}+S_{3}^{2}\right) \geq-12 .
\end{aligned}
$$

We eliminate $d_{3}$ using (5.3) and complete the squares involving $y_{2}$ and $y_{3}$, to find:

$$
\begin{aligned}
-\frac{135}{k^{2}}-4 u_{2}^{2}-6 u_{3}^{2} & +15 M^{2}+\left(\left(S_{2}^{1}\right)^{2}-2 S_{2}^{2}\right)+\left(\frac{2}{3}\left(S_{3}^{1}\right)^{2}-2 S_{3}^{2}\right) \\
+ & \frac{30}{k}\left(2 x_{2}+d_{2}-2 k_{2}+1\right) \geq-12,
\end{aligned}
$$

where

$$
\begin{aligned}
& u_{2}=y_{2}-\frac{1}{2}\left(\frac{9}{k}+S_{2}^{1}\right) \\
& u_{3}=y_{3}-\frac{1}{3}\left(-\frac{9}{k}+S_{3}^{1}\right) .
\end{aligned}
$$

Implementing the second condition in (5.3) we get $2 u_{2}+3 u_{3}=0$. Introduce new variables

$$
\begin{aligned}
& u:=2 u_{2}=-3 u_{3} \\
& x:=2 x_{2}+d_{2}-2 k_{2}+1 .
\end{aligned}
$$

Substituting back into the expressions for $L_{2}$ and $L_{3}$ we get

$$
\begin{aligned}
L_{2}=\frac{9}{k} & \left(e^{\prime}+\zeta^{\prime}\right)+\frac{1}{2}\left(x-d_{2}+2 k_{2}-1\right) f^{\prime} \\
& +\frac{1}{2}\left(u+\frac{9}{k}+S_{2}^{1}\right)\left(n_{1}^{\prime}+o_{2}^{\prime}\right)+3 M \\
L_{3}=- & \frac{6}{k}\left(e^{\prime}+\zeta^{\prime}\right)+\frac{1}{3}\left(-x-d_{3}+3 k_{3}-3\right) f^{\prime} \\
& +\frac{1}{3}\left(-u-\frac{9}{k}+S_{3}^{1}\right)\left(n_{1}^{\prime}+o_{2}^{\prime}\right)-2 M .
\end{aligned}
$$

Similarly for the conditions (\#C2 $\left.f^{\prime}\right)$ and $(\# \mathbf{S} s)$ we get

$$
\frac{5}{3} u^{2}-15 M^{2} \leq 12-\frac{135}{k^{2}}+\frac{30}{k} x+\left(\left(S_{2}^{1}\right)^{2}-2 S_{2}^{2}\right)+\left(\frac{2}{3}\left(S_{3}^{1}\right)^{2}-2 S_{3}^{2}\right),
$$


and

$$
\left(\frac{18}{k}\left(e^{\prime}+\zeta^{\prime}\right)+x f^{\prime}+\left(u+\frac{9}{k}\right)\left(n_{1}^{\prime}+o_{2}^{\prime}\right)+6 M\right) \cdot h^{\prime}<0 .
$$

respectively.

We will use the flexibility we have in choosing $M$ to show that (5.5) and (5.6) have solutions that lead to integral coefficients in (5.4). The key observation here is that since $\operatorname{span}\left(e^{\prime}+\zeta^{\prime}, f^{\prime}, n_{1}^{\prime}+o_{2}^{\prime}\right) \subset H^{2}\left(B^{\prime}, \mathbb{Z}\right)$ contains an ample class, the Hodge index theorem implies that $M^{2} \leq 0$. Therefore, one expects that there will be non-effective admissible $M$ 's which will make (5.6) easier to satisfy.

Note that the means inequality implies that $\left(S_{2}^{1}\right)^{2}-2 S_{2}^{2} \leq 0$ with equality if and only if all the $a_{2 k}$ 's are equal to each other. Similarly $(2 / 3)\left(S_{3}^{1}\right)^{2}-$ $2 S_{3}^{2} \leq 0$ with equality if and only if all the $a_{3 k}$ 's are equal to each other. In particular, for any choice of numbers $u, x, k_{2}, k_{3}, a_{i k}$ which satisfies (5.5), the numbers $u, x, k_{2}, k_{3}$ will satisfy

$$
\frac{5}{3} u^{2} \leq 12+15 M^{2}-\frac{135}{k^{2}}+\frac{30}{k} x
$$

as well.

But from Table 1 we see that $k=2 k_{3}-3 k_{2}>0$ for all admissible values of $k_{2}$ and $k_{3}$. Combined with the fact that $e^{\prime}+\zeta^{\prime}$ is an effective curve this implies

$$
\left(x f^{\prime}+\left(u+\frac{9}{k}\right)\left(n_{1}^{\prime}+o_{2}^{\prime}\right)+6 M\right) \cdot h^{\prime}<0 .
$$

On the other hand $f^{\prime}=n_{j}^{\prime}+o_{j}^{\prime}$ for $j=1,2$ and so $f^{\prime} \cdot h^{\prime}>n_{1}^{\prime} \cdot h^{\prime}$ and $f^{\prime} \cdot h^{\prime}>o_{1}^{\prime} \cdot h^{\prime}$. Thus it suffices to check that

$$
\left(\left(x+u+\frac{9}{k}\right) f^{\prime}+6 M\right) \cdot h^{\prime}<0
$$

To make things more concrete recall that the only conditions that we need to impose on $M$ are that $M$ should be $\tau_{B^{\prime}}^{*}$-invariant and that $M$ should be perpendicular to $\operatorname{span}\left(e^{\prime}+\zeta^{\prime}, f^{\prime}, n_{1}^{\prime}+o_{2}^{\prime}\right)$. From [DOPWa, Table 1] we see that the classes $e_{4}^{\prime}-e_{5}^{\prime}, e_{4}^{\prime}-e_{6}^{\prime}, 3 \ell^{\prime}-2\left(e_{4}^{\prime}+e_{5}^{\prime}+e_{6}^{\prime}\right)-3 e_{7}^{\prime}$ constitute a rational basis of the space of such $M$ 's. Let us choose for example $M$ to be of the form

$$
M=z\left(e_{4}^{\prime}-e_{5}^{\prime}\right)
$$


for some integer $z$. With this choice (5.7) and (5.8) become

$$
\frac{5}{3} u^{2}+30 z^{2}-\frac{30}{k} x+\frac{135}{k^{2}}-12 \leq 0,
$$

and

$$
\left(\left(x+u+\frac{9}{k}\right) f^{\prime}+6 z\left(e_{4}^{\prime}-e_{5}^{\prime}\right)\right) \cdot h^{\prime}<0
$$

respectively.

Consider next the class $\gamma:=(x+u+9 / k) f^{\prime}+6 z\left(e_{4}^{\prime}-e_{5}^{\prime}\right)$. Since the Kähler cone is dual to the Mori cone we know that an ample class $h^{\prime}$ with $\gamma \cdot h^{\prime}<0$ will exist as long as $\gamma$ is not effective. But $\gamma$ satisfies $\gamma \cdot e_{4}^{\prime}=x+u+9 / k-6 z$ and so if $6 z>x+u+9 / k$ we will have $\gamma \cdot e_{4}^{\prime}<0$. Under this assumption we have two alternatives: either $\gamma$ is not effective or $\gamma-e_{4}^{\prime}$ is effective. However we have $\left(\gamma-e_{4}^{\prime}\right) \cdot f^{\prime}=-1$ and $f^{\prime}$ moves, so $\gamma-e_{4}^{\prime}$ and hence $\gamma$ can not be effective.

In other words, as a first check for the consistency of the inequalities (5.5) and (5.6) it suffices to make sure that in the 3-space with coordinates $(x, u, z)$ one can find points between the plane

$$
6 z=x+u+\frac{9}{k}
$$

and the paraboloid

$$
\frac{5}{3} u^{2}+30 z^{2}-\frac{30}{k} x+\frac{135}{k^{2}}-12=0 .
$$

If we use the equation of the plane to eliminate $x$ and substitute the result in (5.9) we obtain the quadratic inequality

$$
\frac{5}{3}\left(u+\frac{9}{k}\right)^{2}+30\left(z-\frac{3}{k}\right)^{2}-12 \leq 0,
$$

which always has solutions regardless of the value of $k$.

To find an actual solution we will choose a particular value for $k$. By examining Table 1 we see that the possible values of $k=2 k_{3}-3 k_{2}$ are $1,2,3$ and 6. Furthermore, since all the coefficients in (5.4) must be integers, $k$ has to divide $\operatorname{gcd}(6,9)=3$ i.e. we may have either $k=1$ (which corresponds to $k_{2}=3$ and $k_{3}=5$ ) or $k=3$ (which corresponds to $k_{2}=3$ and $k_{3}=6$. For concreteness we choose the second case, i.e.

$$
k_{2}=3, \quad k_{3}=6, \quad k=3 .
$$


Note that the geometry of this case has already been carefully analyzed in sections 4.2 and 4.3. In particular we showed that for these values of $k_{i}$ there are spectral pairs $\left(C_{i}, \mathcal{N}_{i}\right)$ which are deformable to smooth pairs and which satisfy (4.1) and (4.2).

To minimize (5.11) we will take

$$
u=-3, \quad z=1, \quad x=5,
$$

where the value of $x$ is chosen to satisfy (5.10).

We then have

$$
\begin{aligned}
& L_{2}=3\left(e^{\prime}+\zeta^{\prime}\right)+\frac{1}{2}\left(4-d_{2}\right) f^{\prime}+\frac{1}{2}\left(6+S_{2}^{1}\right)\left(n_{1}^{\prime}+o_{2}^{\prime}\right)+3\left(e_{4}^{\prime}-e_{5}^{\prime}\right) \\
& L_{3}=-2\left(e^{\prime}+\zeta^{\prime}\right)+\frac{1}{3}\left(16-d_{3}\right) f^{\prime}+\frac{1}{3}\left(-6+S_{3}^{1}\right)\left(n_{1}^{\prime}+o_{2}^{\prime}\right)-2\left(e_{4}^{\prime}-e_{5}^{\prime}\right) .
\end{aligned}
$$

We see that all the coefficients in (5.12) will be integral as long as: $d_{2}$ is even, $d_{3} \equiv 1(\bmod 3), S_{2}^{1}$ is even and $S_{3}^{1}$ is divisible by 3 .

The inequality (5.5) now reads

$$
-2 \leq\left(\left(S_{2}^{1}\right)^{2}-2 S_{2}^{2}\right)+\left(\frac{2}{3}\left(S_{2}^{1}\right)^{2}-2 S_{2}^{2}\right)
$$

and the inequality (5.6) reads

$$
\left(6\left(e^{\prime}+\zeta^{\prime}\right)+5 f^{\prime}+6\left(e_{4}^{\prime}-e_{5}^{\prime}\right)\right) \cdot h^{\prime}<0
$$

Note that (5.14) does not involve the numbers $a_{i k}$ and so all the restrictions on the $a_{i k}$ 's come from (5.13) and from the integrality of (5.12).

In view of the discussion about the means inequality above we see that (5.13) will be automatically satisfied if we take all $a_{2 k}$ 's to be equal to a fixed integer $a_{2} \geq 0$ and all $a_{3 k}$ 's to be equal to another fixed integer $a_{3} \geq 0$. Moreover with such a choice we clearly have $S_{2}^{1}=2 a_{2}$ and $S_{3}^{1}=3 a_{3}$ and so we have infinitely many possibilities for the numbers $a_{i k}$. Since $d_{2}$ and $d_{3}$ are unconstrained except for the conditions $d_{2} \equiv 0(\bmod 2)$ and $d_{3} \equiv 1$ $(\bmod 3)$ we see that all the conditions (S), (I) and (C1-3) will be satisfied if we can prove the following:

Claim 5.5. There exists an ample class $h^{\prime} \in \operatorname{Pic}\left(B^{\prime}\right)$ satisfying (5.14).

Proof. As explained above the existence of $h^{\prime}$ is equivalent to showing that the class

$$
6\left(e^{\prime}+\zeta^{\prime}\right)+5 f^{\prime}+6\left(e_{4}^{\prime}-e_{5}^{\prime}\right)=6\left(e_{1}^{\prime}+e_{9}^{\prime}\right)+5 f^{\prime}+6\left(e_{4}^{\prime}-e_{5}^{\prime}\right)
$$


is not in the Mori cone of $B^{\prime}$. First consider the class $\xi^{\prime}:=e_{4}^{\prime}-e_{5}^{\prime}+e_{9}^{\prime}+f^{\prime} \in$ $\operatorname{Pic}\left(B^{\prime}\right)$. We have $\xi^{\prime 2}=-1$ and $\xi \cdot f^{\prime}=1$. So $\xi^{\prime}$ is an exceptional class on $B^{\prime}$. It is well known [DPT80] that on a general rational elliptic surface every exceptional class is effective and is a section. Since our $B^{\prime}$ is not generic we can't use this statement to conclude that $\xi^{\prime}$ is the class of a section. However we have

Lemma 5.6. The divisor $\xi^{\prime}$ satisfies

$$
\mathcal{O}_{B^{\prime}}\left(\xi^{\prime}\right)=c_{1}\left(\left[e_{4}^{\prime}\right]-\left[e_{5}^{\prime}\right]\right) .
$$

In particular $\xi^{\prime}$ is effective and is a section of $\beta^{\prime}: B^{\prime} \rightarrow \mathbb{P}^{1}$.

Proof. Let $\xi^{\prime}$ be the section of $B^{\prime}$ for which $\left[\xi^{\prime}\right]=\left[e_{4}^{\prime}\right]-\left[e_{5}^{\prime}\right]$, that is $c_{1}\left(\left[e_{4}^{\prime}\right]-\left[e_{5}^{\prime}\right]\right)=\overline{\mathcal{O}}_{B^{\prime}}\left(\xi^{\prime}\right)$. Since the group law on the general fiber $f_{t}^{\prime}, t \in \mathbb{P}^{1}$ is defined in terms of the Abel-Jacobi map and since we have taken $e_{9}^{\prime}(t) \in f_{t}^{\prime}$ to be the neutral element for the group law it follows that

$$
\mathcal{O}_{B^{\prime}}\left(\underline{\xi}^{\prime}-e_{9}^{\prime}\right)_{\mid f_{t}^{\prime}}=\left(c_{1}\left(\left[e_{4}^{\prime}\right]-\left[e_{5}^{\prime}\right]\right) \otimes \mathcal{O}_{B^{\prime}}\left(-e_{9}^{\prime}\right)\right)_{\mid f_{t}^{\prime}}=\mathcal{O}_{B^{\prime}}\left(e_{4}^{\prime}-e_{5}^{\prime}\right)_{\mid f_{t}^{\prime}}
$$

for the general $t \in \mathbb{P}^{1}$. Therefore the line bundle $\mathcal{O}_{B^{\prime}}\left(\xi^{\prime}+e_{5}^{\prime}-e_{4}^{\prime}-e_{9}^{\prime}\right)$ must be a combination of vertical divisors on $B^{\prime}$, i.e. we can write

$$
\underline{\xi}^{\prime}=e_{4}^{\prime}-e_{5}^{\prime}+e_{9}^{\prime}+a \cdot f^{\prime}+b \cdot n_{1}+c \cdot n_{2}
$$

for some integers $a, b$ and $c$. By [DOPWa, Formula (4.2)] we have $e_{4}^{\prime} \cdot n_{i}^{\prime}=$ $e_{5}^{\prime} \cdot n_{i}^{\prime}=0$ and $e_{9}^{\prime} \cdot n_{i}^{\prime}=1$ for $i=1,2$. Consider the $I_{2}$ fiber $n_{1}^{\prime} \cup o_{1}^{\prime}$ of $B^{\prime}$. The smooth part $\left(n_{1}^{\prime} \cup o_{1}^{\prime}\right)^{\sharp}:=\left(n_{1}^{\prime} \cup o_{1}^{\prime}\right)-\left(n_{1}^{\prime} \cap o_{1}^{\prime}\right)$ of this fiber is an abelian group isomorphic to $\mathbb{Z} / 2 \times \mathbb{C}^{\times}$with $n_{1}^{\prime}-\left(n_{1}^{\prime} \cap o_{1}^{\prime}\right)$ being the connected component of the identity. By definition the section $\xi^{\prime}$ intersects $n_{1}^{\prime} \cup o_{1}^{\prime}$ at a point which is the difference of the points $e_{4}^{\prime} \cdot\left(n_{1}^{\prime} \cup o_{1}^{\prime}\right)=e_{4}^{\prime} \cdot o_{1}^{\prime}$ and $e_{5}^{\prime} \cdot\left(n_{1}^{\prime} \cup o_{1}^{\prime}\right)=e_{5}^{\prime} \cdot o_{1}^{\prime}$ in the group law of $\left(n_{1}^{\prime} \cup o_{1}^{\prime}\right)^{\sharp}$. Since these two points belong to the same component of $\left(n_{1}^{\prime} \cup o_{1}^{\prime}\right)^{\sharp}$ and the group of connected components of $\left(n_{1}^{\prime} \cup o_{1}^{\prime}\right)^{\sharp}$ is $\mathbb{Z} / 2$ it follows that $\underline{\xi}^{\prime}$ intersects $n_{1}^{\prime} \cup o_{1}^{\prime}$ at a point in $n_{1}^{\prime}$, i.e. $\underline{\xi}^{\prime} \cdot n_{1}^{\prime}=1$. Similarly $\delta^{\prime} \cdot n_{2}^{\prime}=1$. Therefore, intersecting both sides of (5.15) with $n_{1}^{\prime}$ and $n_{2}^{\prime}$ we get $1=1+b$ and $1=1+c$ respectively. Thus $b=c=0$. Finally from the fact that $\underline{\xi}^{\prime 2}=-1$ we compute that $a=1$ and so $\underline{\xi}^{\prime}=\xi^{\prime}$. The lemma is proven.

In view of the previous lemma we have a section $\xi^{\prime}$ of $\beta^{\prime}: B^{\prime} \rightarrow \mathbb{P}^{1}$ and we need to show that the class

$$
\mu:=6 e_{1}^{\prime}+6 \xi^{\prime}-f^{\prime} \in \operatorname{Pic}\left(B^{\prime}\right)
$$

is not in the Mori cone of $B^{\prime}$. 
Assume that $\mu$ is in the Mori cone. Note that by the definition of $\xi^{\prime}$ we have $e_{1}^{\prime} \cdot f^{\prime}=\xi^{\prime} \cdot f^{\prime}=1, e_{1}^{\prime 2}=\xi^{\prime 2}=-1$ and $e_{1}^{\prime} \cdot \xi^{\prime}=1$. Then $\mu \cdot \xi^{\prime}=-1$ and so $\mu-\xi^{\prime}=6 e_{1}^{\prime}+5 \xi^{\prime}-f^{\prime}$ will also have to be in the Mori cone. But now $\left(\mu-\xi^{\prime}\right) \cdot e_{1}^{\prime}=-2$ and so $\mu-2 e_{1}^{\prime}-\xi^{\prime}$ will be in the Mori cone. Intersecting with $\xi^{\prime}$ again we get $\left(\mu-2 e_{1}^{\prime}-\xi^{\prime}\right) \cdot \xi^{\prime}=-2$ and so continuing iteratively we conclude that $-\xi-f$ must be in the Mori cone which is obviously false. This shows that $\mu$ is not in the Mori cone of $B^{\prime}$ and so $h^{\prime}$ ought to exist.

For completeness we will identify an explicit ample class $h^{\prime}$ on $B^{\prime}$ with $\mu \cdot h^{\prime}<0$. We will look for $h^{\prime}$ of the form

$$
h^{\prime}=a f^{\prime}+b e_{1}^{\prime}+c \xi^{\prime}
$$

and will try to adjust the coefficients $a, b$ and $c$ so that $h^{\prime}$ is ample and $\mu \cdot h^{\prime}<0$. First we have the following

Lemma 5.7. The divisor class $h^{\prime}=a f^{\prime}+b e_{1}^{\prime}+c \xi^{\prime}$ is ample provided that $a, b$ and $c$ are positive and $a>|b-c|$.

Proof. Assume that $a, b$ and $c$ are positive and $a>|b-c|$. By the Nakai-Moishezon criterion for ampleness [Har77, Theorem 1.10], $h^{\prime}$ will be ample if $h^{\prime 2}>0$ and if $h^{\prime} \cdot C>0$ for every irreducible curve $C \subset B^{\prime}$.

Let $C \subset B^{\prime}$ be an irreducible curve. Then we have two possibilities: either $C$ is a component of a fiber of $\beta^{\prime}: B^{\prime} \rightarrow \mathbb{P}^{1}$, or $\beta^{\prime}: C \rightarrow \mathbb{P}^{1}$ is a finite map. If $\beta^{\prime}: C \rightarrow \mathbb{P}^{1}$ is finite and $C \neq \xi^{\prime}, e_{1}^{\prime}$, then $C \cdot f^{\prime}>0, C \cdot e_{1}^{\prime} \geq 0$ and $C \cdot \xi^{\prime} \geq 0$. In particular the fact that $a, b$ and $c$ are positive implies that $C \cdot h^{\prime}>0$. Hence $h^{\prime}$ will be ample if we can show that the intersections $h^{\prime 2}$, $h^{\prime} \cdot f^{\prime}, h^{\prime} \cdot n_{i}^{\prime}, h^{\prime} \cdot o_{i}^{\prime}, h^{\prime} \cdot e_{1}^{\prime}$ and $h^{\prime} \cdot \xi^{\prime}$ are all positive. For this we calculate

$$
\begin{aligned}
h^{\prime} \cdot e_{1}^{\prime} & =a+c-b \\
h^{\prime} \cdot \xi^{\prime} & =a+b-c \\
h^{\prime} \cdot f^{\prime} & =b+c \\
h^{\prime} \cdot n_{1}^{\prime} & =h^{\prime} \cdot n_{2}^{\prime}=c \\
h^{\prime} \cdot o_{1}^{\prime} & =h^{\prime} \cdot o_{2}^{\prime}=b \\
h^{\prime 2} & =-b^{2}-c^{2}+2 a b+2 a c+2 b c=b(a+c-b)+c(a+b-c)+a b+a c,
\end{aligned}
$$

which are manifestly positive provided that $a>|b-c|$. The lemma is proven.

Now we see that the condition $\mu \cdot h^{\prime}<0$ translates into $12 a<b+c$, i.e we may take $h^{\prime}=25 f^{\prime}+144 e_{1}^{\prime}+168 \xi^{\prime}$. This finishes the proof of the claim. 


\section{Summary of the construction}

In this section we recapitulate the main points of the construction. Recall that we want to build a quadruple $\left(X, H, \tau_{X}, V\right)$ satisfying:

$(\mathbb{Z} / 2) X$ is a smooth Calabi-Yau 3-fold and $\tau_{X}: X \rightarrow X$ is a freely acting involution. $H$ is a fixed Kähler structure (ample line bundle) on $X$

(S) $V$ is an $H$-stable vector bundle of rank five on $X$.

(I) $V$ is $\tau_{X}$-invariant.

(C1) $c_{1}(V)=0$.

(C2) $c_{2}(X)-c_{2}(V)$ is effective.

(C3) $c_{3}(V)=12$.

The construction is carried out in several steps.

\subsection{The construction of $\left(X, \tau_{X}\right)$}

$X$ is built as the fiber product of two rational elliptic surfaces of special type.

6.1.1. Building special rational elliptic surfaces. Let $\Gamma_{1} \subset \mathbb{P}^{2}$ be a nodal cubic with a node $A_{8}$. Choose four generic points on $\Gamma_{1}$ and label them $A_{1}, A_{2}, A_{3}, A_{7}$. Let $\Gamma \subset \mathbb{P}^{2}$ be the unique smooth cubic which passes trough $A_{1}, A_{2}, A_{3}, A_{7}, A_{8}$ and is tangent to the line $\left\langle A_{7} A_{i}\right\rangle$ for $i=1,2,3$ and 8. Consider the pencil of cubics spanned by $\Gamma_{1}$ and $\Gamma$. All cubics in this pencil pass trough $A_{1}, A_{2}, A_{3}, A_{7}, A_{8}$ and are tangent to $\Gamma$ at $A_{8}$. Let $A_{4}, A_{5}, A_{6}$ be the remaining three base points, and let $B$ denote the blow-up of $\mathbb{P}^{2}$ at the points $A_{i}, i=1,2, \ldots, 8$ and the point $A_{9}$ which is infinitesimally near $A_{8}$ and corresponds to the line $\left\langle A_{7} A_{8}\right\rangle$.

The pencil becomes the anti-canonical map $\beta: B \rightarrow \mathbb{P}^{1}$ which is an elliptic fibration with a section. The map $\beta$ has two reducible fibers $f_{i}=$ $n_{i} \cup o_{i}, i=1,2$ of type $I_{2}$. We denote by $e_{i}, i=1, \ldots, 7$ and $e_{9}$ the exceptional divisors corresponding to $A_{i}, i=1, \ldots, 7$ and $A_{9}$, and by $e_{8}$ the reducible divisor $e_{9}+n_{1}$. The divisors $e_{i}$ together with the pullback $\ell$ of a class of a line from $\mathbb{P}^{2}$ form a standard basis in $H^{2}(B, \mathbb{Z})$. 
The surface $B$ has an involution $\alpha_{B}$ which is uniquely characterized by the properties: $\alpha_{B}$ commutes with $\beta, \alpha_{B}$ induces an involution on $\mathbb{P}^{1}$, and $\alpha_{B}$ fixes the proper transform of $\Gamma$ pointwise.

Choosing $e_{9}$ as the zero section of $\beta$, we can interpret any other section $\xi$ as an automorphism $t_{\xi}: B \rightarrow B$ which acts along the fibers of $\beta$. The automorphism $\tau_{B}=t_{e_{1}} \circ \alpha_{B}$ is again an involution of $B$ which commutes with $\beta$, induces the same involution on $\mathbb{P}^{1}$ as $\alpha_{B}$ and has four isolated fixed points sitting on the same fiber of $\beta$.

The special rational elliptic surfaces form a four dimensional irreducible family. Their geometry was the subject of [DOPWa].

6.1.2. Building $\left(X, \tau_{X}\right)$. Choose two special rational elliptic surfaces $\beta: B \rightarrow \mathbb{P}^{1}$ and $\beta^{\prime}: B^{\prime} \rightarrow \mathbb{P}^{1}$ so that the discriminant loci of $\beta$ and $\beta^{\prime}$ in $\mathbb{P}^{1}$ are disjoint, $\alpha_{B}$ and $\alpha_{B^{\prime}}$ induce the same involution on $\mathbb{P}^{1}$ and the fix loci of $\tau_{B}$ and $\tau_{B^{\prime}}$ sit over different points in $\mathbb{P}^{1}$. The fiber product $X:=B \times_{\mathbb{P}^{1}} B^{\prime}$ is a smooth Calabi-Yau 3-fold which is elliptic and has a freely acting involution $\tau_{B} \times \tau_{B^{\prime}}$ and another (non-free) involution $\alpha_{X}:=\alpha_{B} \times \alpha_{B^{\prime}}$. For concreteness we fix the elliptic fibration of $X$ to be the projection $\pi: X \rightarrow B^{\prime}$ to $B^{\prime}$.

The Calabi-Yau's form a nine dimensional irreducible family.

6.1.3. Building $H$. Choose any ample divisor $H_{0}$ on $X$ and take $H=$ $H_{0}+n \cdot \pi^{*} h^{\prime}$ for some positive integer $n$. Then the divisor $H$ will be ample as long as $h^{\prime}$ is ample on $B^{\prime}$ and $n \gg 0$.

Choose $h^{\prime}=25 f^{\prime}+144 e_{1}^{\prime}+168 \xi^{\prime}$ with $\xi^{\prime}$ being the unique section of $\beta^{\prime}: \mathbb{P}^{1} \rightarrow B^{\prime}$ satisfying $\left[e_{4}^{\prime}\right]-\left[e_{5}\right]^{\prime}=\left[\xi^{\prime}\right]$. The divisor class $h^{\prime} \in \operatorname{Pic}\left(B^{\prime}\right)$ is ample on $B^{\prime}$ by Lemma 5.7.

\subsection{The construction of $V$}

The bundle $V$ is build as a non-split extension

$$
0 \rightarrow V_{2} \rightarrow V \rightarrow V_{3} \rightarrow 0
$$

of two $\tau_{X}$-invariant stable vector bundles $V_{2}$ and $V_{3}$ of ranks 2 and 3 respectively.

Each $V_{i}$ is constructed via the spectral cover construction on $X$.

6.2.1. Building $V_{2}$ and $V_{3}$. Choose curves $\bar{C}_{2}, C_{3} \subset B$, so that 
- $\bar{C}_{2} \in\left|\mathcal{O}_{B}\left(2 e_{9}+2 f\right)\right|$ and $C_{3} \in\left|\mathcal{O}_{B}\left(3 e_{9}+6 f\right)\right|$.

- $\bar{C}_{2}, C_{3}$ are $\alpha_{B}$-invariant.

- $\bar{C}_{2}$ and $C_{3}$ are smooth and irreducible.

Set $C_{2}:=\bar{C}_{2}+f_{\infty}$ where $f_{\infty}$ is the smooth fiber of $\beta$ containing the fixed points of $\tau_{B}$.

The space of such $\bar{C}_{2}$ 's is an open set (see section 4$)$ in $\mathbb{P}\left(H^{0}\left(B, \mathcal{O}_{B}\left(2 e_{9}+\right.\right.\right.$ $2 f))^{+}$) where $H^{0}\left(B, \mathcal{O}_{B}\left(2 e_{9}+2 f\right)\right)^{ \pm}$denote the spaces of invariants/antiinvariants for the $\alpha_{B}$ action on $H^{0}\left(B, \mathcal{O}_{B}\left(2 e_{9}+2 f\right)\right.$. Using the explicit equations (4.3) of the spectral curves we easily that all such $C_{2}$ form a 2 dimensional irreducible family. The space of permissible $C_{3}$ 's is an open subset in the disjoint union of the projective spaces $\mathbb{P}\left(H^{0}\left(B, \mathcal{O}_{B}\left(3 e_{9}+6 f\right)\right)^{ \pm}\right)$, which have dimensions 8 and 6 .

Fix an even integer $d_{2}$ and an integer $d_{3}$ satisfying $d_{3} \equiv 1(\bmod 3)$. Choose line bundles $\mathcal{N}_{2}$ and $\mathcal{N}_{3}$ on $C_{2}$ and $C_{3}$ respectively, which satisfy

- $\mathcal{N}_{i} \in \operatorname{Pic}^{d_{i}}\left(C_{i}\right)$ for $i=1,2$,

- $\mathcal{N}_{i}=\boldsymbol{T}_{C_{i}}\left(\mathcal{N}_{i}\right):=\alpha_{B \mid C_{i}}^{*} \mathcal{N}_{i} \otimes \mathcal{O}_{C_{i}}\left(e_{1}-e_{9}+f\right)$ for $i=1,2$.

As explained in sections 4.2 and 4.3 , such $\mathcal{N}_{2}$ 's and $\mathcal{N}_{3}$ 's are parameterized by abelian subvarieties of $\mathrm{Pic}^{d_{2}-1}\left(\bar{C}_{2}\right)$ and $\mathrm{Pic}^{d_{3}}\left(C_{3}\right)$ of dimensions equal to the genera of the quotient curves $\bar{C}_{2} / \alpha_{\bar{C}_{2}}$ and $C_{3} / \alpha_{C_{3}}$ respectively. Thus there is a one dimensional space of $\mathcal{N}_{2}$ 's and a six dimensional space of $\mathcal{N}_{3}$ 's.

Let $\Sigma_{i}=C_{i} \times_{\mathbb{P}^{1}} B^{\prime}$ for $i=1,2$. Recall that $\beta^{\prime}: B^{\prime} \rightarrow \mathbb{P}^{1}$ has two $I_{2}$ fibers $f_{1}^{\prime}$ and $f_{2}^{\prime}$. Let $F_{1}, F_{2}$ be the corresponding (smooth) fibers of $\beta: B \rightarrow \mathbb{P}^{1}$. Let $C_{i} \cap F_{j}=\left\{p_{i j k}\right\}_{k=1}^{i}$ for $i=2,3, j=1,2$. Then $\Sigma_{i} \rightarrow C_{i}$ is an elliptic surface having $2 i$ fibers of type $I_{2}:\left\{p_{i j k}\right\} \times f_{j}^{\prime}$. Also $\Sigma_{i} \subset X$ and the natural projection $\pi_{\mid \Sigma_{i}}: \Sigma_{i} \rightarrow B^{\prime}$ is finite of degree $i$.

Fix non-negative integers $a_{2}$ and $a_{3}$. Define

$$
\begin{gathered}
V_{i}= \\
\boldsymbol{F} \boldsymbol{M}_{X}\left(\left(\Sigma_{i},\left(\pi_{\mid \Sigma_{i}}\right)^{*} \mathcal{N}_{i} \otimes \mathcal{O}_{\Sigma_{i}}\left(-a_{i} \sum_{k=1}^{i}\left(\left\{p_{i 1 k}\right\} \times n_{1}^{\prime}+\left\{p_{i 2 k}\right\} \times o_{2}^{\prime}\right)\right)\right)\right) \otimes \pi^{*} L_{i},
\end{gathered}
$$

where $L_{2}$ and $L_{3}$ are the line bundles

$$
\begin{aligned}
& L_{2}=3\left(e_{1}^{\prime}+e_{4}^{\prime}-e_{5}^{\prime}+e_{9}^{\prime}\right)+\frac{1}{4}\left(4-d_{2}\right) f^{\prime}+\left(3-a_{2}\right)\left(n_{1}^{\prime}+o_{2}^{\prime}\right) \\
& L_{3}=-2\left(e_{1}^{\prime}+e_{4}^{\prime}-e_{5}^{\prime}+e_{9}^{\prime}\right)+\frac{1}{3}\left(16-d_{3}\right) f^{\prime}+\left(-2+a_{3}\right)\left(n_{1}^{\prime}+o_{2}^{\prime}\right),
\end{aligned}
$$


on $B^{\prime}$.

6.2.2. Building $V$. Take $V$ to be a non-split extension of $V_{2}$ by $V_{3}$ which is $\tau_{X}$-invariant. As explained in section 5.2, the space of all such extensions of $V_{2}$ by $V_{3}$ is the union of projective spaces $\mathbb{P}\left(H^{1}\left(X, V_{3}^{\vee} \otimes V_{2}\right)^{+}\right) \cup \mathbb{P}\left(H^{1}\left(X, V_{3}^{\vee} \otimes\right.\right.$ $\left.\left.V_{2}\right)^{-}\right)$where $H^{1}\left(X, V_{3}^{\vee} \otimes V_{2}\right)^{ \pm}$denote the invariants/anti-invariants for the $\tau_{X}$ action on $H^{1}\left(X, V_{3}^{\vee} \otimes V_{2}\right)$.

Furthermore, it is shown in section 5.2 that

$$
\operatorname{dim} H^{1}\left(X, V_{3}^{\vee} \otimes V_{2}\right) \geq\left(\left((-1)_{B}^{*} C_{3}\right) \cdot C_{2}\right) \cdot\left(L_{2}^{\prime} \cdot f^{\prime}-L_{3}^{\prime} \cdot f^{\prime}\right)=150,
$$

and from the explicit description of $H^{1}\left(X, V_{3}^{\vee} \otimes V_{2}\right)$ we see that the \pm decomposition breaks this as $150=70+80$, so the dimension of the admissible extensions of $V_{2}$ by $V_{3}$ is at least 79 .

In other words, for a fixed $\left(X, \tau_{X}, H\right)$ as above we find infinitely many components of the moduli space of $V$ 's satisfying $(\mathbf{S}),(\mathbf{I})$ and (C1-3). Each component corresponds to a choice of the integers $a_{2}, a_{3}, d_{2}$ and $d_{3}$ and has dimension $2+8+1+6+79=96$.

\section{Appendix A Hecke transforms}

In this appendix we review the definition and some basic properties of the Hecke transforms (aka 'elementary modifications') of vector bundles along divisors. For more details the reader may wish to consult [Mar82, Mar87], [Fri98].

\section{A.1 Definition and basic properties}

Let $X$ be a smooth complex projective variety. Let $\imath: D \hookrightarrow X$ be a divisor with normal crossings.

Let $E \rightarrow X$ be a vector bundle and let $(\xi)$ be a short exact sequence of vector bundles on $D$ of the form

$$
\text { ( } \xi) \quad: \quad 0 \longrightarrow F \longrightarrow E_{\mid D} \longrightarrow G \longrightarrow 0 .
$$

There are two Hecke transforms $\boldsymbol{H e c k e}_{(\xi)}^{ \pm}(E)$ attached to the pair $(E,(\xi))$. 


\section{Definition A.1.}

(i) The down-Hecke transform of $E$ along $(\xi)$ is the coherent sheaf Hecke $_{(\xi)}^{-}(E)$ defined by the exact sequence

$$
0 \longrightarrow \operatorname{Hecke}_{(\xi)}^{-}(E) \longrightarrow E \longrightarrow \imath_{*} G \longrightarrow 0 .
$$

(ii) The up-Hecke transform of $E$ along $(\xi)$ is the coherent sheaf

$$
\boldsymbol{H e c k e}_{(\xi)}^{+}(E)=\left(\boldsymbol{H e c k e}_{\left(\xi^{\vee}\right)}^{-}\left(E^{\vee}\right)\right)^{\vee}
$$

The first properties of the Hecke transforms are given by the following two lemmas.

\section{Lemma A.2.}

(a) The sheaves $\boldsymbol{H e c k e}_{(\xi)}^{+}(E)$ and $\boldsymbol{H e c k e}_{(\xi)}^{-}(E)$ are locally free.

(b) The up-Hecke transform $\boldsymbol{H e c k e}_{(\xi)}^{+}(E)$ of $E$ along $(\xi)$ fits in the exact sequence

$$
0 \longrightarrow E \longrightarrow \operatorname{Hecke}_{(\xi)}^{+}(E) \longrightarrow \imath_{*} F \otimes \mathcal{O}_{X}(D) \longrightarrow 0
$$

(c) $\operatorname{Hecke}_{(\xi)}^{+}(E)_{\mid D}$ and $\boldsymbol{H e c k e}_{(\xi)}^{-}(E)_{\mid D}$ are furnished with natural exact sequences

$$
\begin{gathered}
\left(\xi^{-}\right) \quad: \quad 0 \longrightarrow G(-D) \longrightarrow \text { Hecke }_{(\xi)}^{-}(E)_{\mid D} \longrightarrow F \longrightarrow 0 \\
\left(\xi^{+}\right) \quad: \quad 0 \longrightarrow G \longrightarrow \text { Hecke }_{(\xi)}^{+}(E)_{\mid D} \longrightarrow F(D) \longrightarrow 0
\end{gathered}
$$

(d) $\operatorname{Hecke}_{(\bullet)}^{+}(\bullet)$ and $\boldsymbol{H e c k e}_{(\bullet)}^{-}(\bullet)$ are mutually inverse in the sense that

$$
\boldsymbol{H e c k e}_{\left(\xi^{-}\right)}^{+}\left(\boldsymbol{H e c k e}_{(\xi)}^{-}(E)\right)=E, \quad \operatorname{Hecke}_{\left(\xi^{+}\right)}^{-}\left(\boldsymbol{H e c k e} \boldsymbol{( \xi )}_{(\xi)}^{+}(E)\right)=E .
$$

(e) $\operatorname{Hecke}_{(\xi)}^{-}(E)=\operatorname{Hecke}_{(\xi)}^{+}(E)(-D)$. 
Proof. The proof of (a) is straightforward. For the proof of (b) recall that by definition the dual bundle $\boldsymbol{H e c k e}_{(\xi)}^{+}(E)^{\vee}$ fits in the short exact sequence of sheaves

$$
0 \longrightarrow \boldsymbol{H e c k e}_{(\xi)}^{+}(E)^{\vee} \longrightarrow E^{\vee} \longrightarrow \imath_{*}\left(F^{\vee}\right) \longrightarrow 0 .
$$

Application of $\mathcal{H o m}_{\mathcal{O}_{X}}\left(\bullet, \mathcal{O}_{X}\right)$ combined with the fact that $\imath_{*}\left(F^{\vee}\right)$ is torsion yields the long exact sequence

$$
\begin{aligned}
& 0 \rightarrow \mathcal{H o m}_{\mathcal{O}_{X}}\left(E^{\vee}, \mathcal{O}_{X}\right) \rightarrow \mathcal{H o m}_{\mathcal{O}_{X}}\left(\boldsymbol{H e c k e}_{(\xi)}^{+}(E)^{\vee}, \mathcal{O}_{X}\right) \rightarrow \\
& \rightarrow \mathcal{E} x t_{\mathcal{O}_{X}}^{1}\left(\imath_{*}\left(F^{\vee}\right), \mathcal{O}_{X}\right) \rightarrow \mathcal{E} t_{\mathcal{O}_{X}}^{1}\left(E^{\vee}, \mathcal{O}_{X}\right) \quad \rightarrow \ldots
\end{aligned}
$$

Furthermore $E^{\vee}$ and $\mathcal{O}_{X}$ are both locally free and hence every extension of $E^{\vee}$ by $\mathcal{O}_{X}$ splits locally yielding $\mathcal{E} x t_{\mathcal{O}_{X}}^{1}\left(E^{\vee}, \mathcal{O}_{X}\right)=0$. Thus we obtain the exact sequence

$$
0 \longrightarrow E \longrightarrow \boldsymbol{H e c k e}_{(\xi)}^{+}(E) \longrightarrow \mathcal{E} x t_{\mathcal{O}_{X}}^{1}\left(\imath_{*}\left(F^{\vee}\right), \mathcal{O}_{X}\right) \longrightarrow 0 .
$$

To calculate $\mathcal{E} x t_{\mathcal{O}_{X}}^{1}\left(\imath_{*}\left(F^{\vee}\right), \mathcal{O}_{X}\right)$ consider the ideal sequence of the divisor $D$ :

$$
0 \longrightarrow \mathcal{O}_{X} \longrightarrow \mathcal{O}_{X}(D) \longrightarrow \mathcal{O}_{D}(D) \longrightarrow 0 .
$$

After apllying $\mathcal{H o m}_{\mathcal{O}_{X}}\left(\imath_{*}\left(F^{\vee}\right), \bullet\right)$ and taking into account that $\mathcal{O}_{X}$ and $\mathcal{O}_{X}(D)$ are locally free sheaves, we get the exact sequence

$$
\begin{aligned}
0 & \longrightarrow \mathcal{H o m}_{\mathcal{O}_{X}}\left(\imath_{*}\left(F^{\vee}\right), \mathcal{O}_{D} D\right) \longrightarrow \mathcal{E x t}_{\mathcal{O}_{X}}^{1}\left(\imath_{*}\left(F^{\vee}\right), \mathcal{O}_{X}\right) \longrightarrow \\
& \longrightarrow \mathcal{E x t}_{\mathcal{O}_{X}}^{1}\left(\imath_{*}\left(F^{\vee}\right), \mathcal{O}_{X}(D)\right) \longrightarrow \ldots
\end{aligned}
$$

To understand the map

$$
\mathcal{E} x t_{\mathcal{O}_{X}}^{1}\left(\imath_{*}\left(F^{\vee}\right), \mathcal{O}_{X}\right) \longrightarrow \mathcal{E} x t_{\mathcal{O}_{X}}^{1}\left(\imath_{*}\left(F^{\vee}\right), \mathcal{O}_{X}(D)\right),
$$

consider a point $p \in D \subset X$. Let $R:=\mathcal{O}_{X, p}$ and let $t \in R$ be a local equation of $D$ around $p$. Let $M$ be the finitely generated $R / t R$ module whose sheafification gives $F^{\vee} \rightarrow D$ in a neighborhood of $p$.

An element $(\alpha) \in \mathcal{E}_{x} t_{\mathcal{O}_{X}}^{1}\left(\imath_{*}\left(F^{\vee}\right), \mathcal{O}_{X}\right)_{p}$ of the stalk of $\mathcal{E} x t_{\mathcal{O}_{X}}^{1}\left(\imath_{*}\left(F^{\vee}\right), \mathcal{O}_{X}\right)$ at $p$ is an extension of $R$-modules of the form

$$
(\alpha) \quad: \quad 0 \longrightarrow R \longrightarrow A \longrightarrow M \longrightarrow 0,
$$

where $M$ is given its $R$-module structure via $R \rightarrow R / t R$. 
The image $(\beta) \in \mathcal{E} x t_{\mathcal{O}_{X}}^{1}\left(\imath_{*}\left(F^{\vee}\right), \mathcal{O}_{X}(D)\right)_{p}$ of $(\alpha)$ under the map (A.1) is just the pushout of the extension $(\alpha)$ via the homomorphism

$$
R \rightarrow \frac{1}{t} R \text {. }
$$

That is, there is a commutative diagram

$(\alpha)$

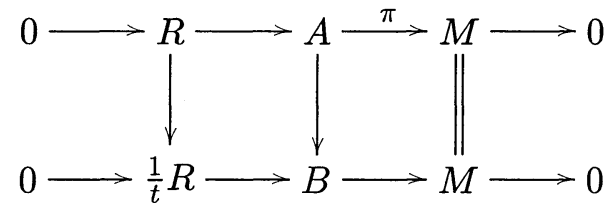

and $B=\left(A \oplus \frac{1}{t} R\right) / R$.

On the other hand, since $t R$ annihilates $M$ we have $\pi(t x)=t \pi(x)=0$, for all $x \in A$. In particular the map

$$
\begin{aligned}
s: & A \oplus \frac{1}{t} R \longrightarrow \frac{1}{t} R \\
x \oplus \frac{f}{t} & \longrightarrow \frac{t x}{t}+\frac{f}{t},
\end{aligned}
$$

is well defined and descends to $B$ to a map splitting the exact sequence

$$
0 \longrightarrow \frac{1}{t} R \longrightarrow B \longrightarrow M \longrightarrow 0 .
$$

Therefore the map

$$
\mathcal{E} x t_{\mathcal{O}_{X}}^{1}\left(\imath_{*}\left(F^{\vee}\right), \mathcal{O}_{X}\right) \longrightarrow \mathcal{E} x t_{\mathcal{O}_{X}}^{1}\left(\imath_{*}\left(F^{\vee}\right), \mathcal{O}_{X}(D)\right)
$$

is the zero map and we get an isomorphism

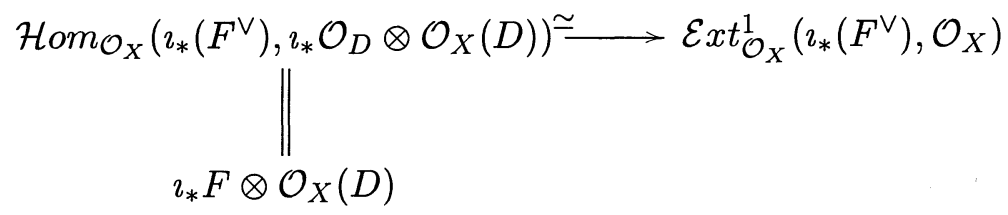

which concludes the proof of the lemma.

There is a natural symmetry between the up and down Hecke transforms. If $X, D, E$ and $(\xi)$ are as above, then we can form the dual exact sequence

$$
\left(\xi^{\vee}\right) \quad: \quad 0 \longrightarrow G^{\vee} \longrightarrow E_{\mid D}^{\vee} \longrightarrow F^{\vee} \longrightarrow 0,
$$

and the up and down Hecke transforms of $E^{\vee}$ along $\left(\xi^{\vee}\right)$. The relation with the Hecke transforms of $E$ is given by the following lemma. 


\section{Lemma A.3.}

$$
\begin{aligned}
& \boldsymbol{H e c k e}_{(\xi)}^{+}(E)^{\vee} \simeq \boldsymbol{H e c k e}_{\left(\xi^{\vee}\right)}^{-}\left(E^{\vee}\right) \\
& \boldsymbol{H e c k e}_{(\xi)}^{-}(E)^{\vee} \simeq \boldsymbol{H e c k e}_{\left(\xi^{\vee}\right)}^{+}\left(E^{\vee}\right)
\end{aligned}
$$

Proof. Clear.

\section{A.2 Geometric interpretation - flips}

Let $(E, \xi)$ be as in Section A.1 and let $\tau \rightarrow \mathbb{P}(E)$ be the relatively ample tautological line bundle. Denote by $Y:=\mathrm{Bl}_{\mathbb{P}(F)} \mathbb{P}(E)$ the blow-up of $\mathbb{P}(E)$ along $\mathbb{P}(F)$.

Let $p: Y \rightarrow \mathbb{P}(E)$ be the blow-up morphism and let $\mathcal{E} \subset Y$ be the exceptional divisor. The image of $Y$ under the full linear system $p^{*} \tau \otimes$ $\mathcal{O}_{Y}(-\mathcal{E})$ is again a projective bundle $\mathbb{P}\left(E^{\prime}\right) \rightarrow X$. We have the following diagram

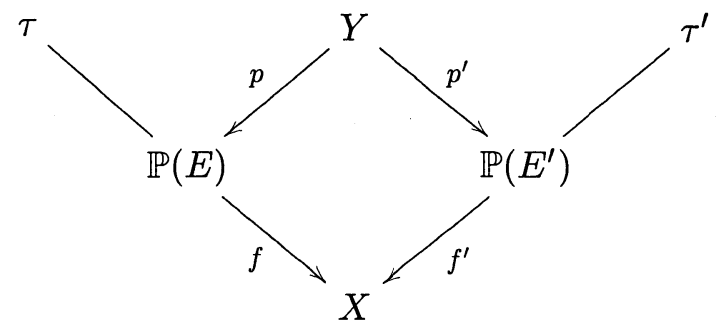

where $\tau \rightarrow \mathbb{P}(E)$ and $\tau^{\prime} \rightarrow \mathbb{P}\left(E^{\prime}\right)$ are relatively ample line bundles having the properties

$$
\begin{aligned}
f_{*} \tau & =E^{\vee} \\
f_{*}^{\prime} \tau^{\prime} & =E^{\prime \vee} \\
p^{\prime *} \tau^{\prime} & =p^{*} \tau \otimes \mathcal{O}_{Y}(-\mathcal{E})
\end{aligned}
$$

To identify $E^{\prime}$ in terms of Hecke transforms consider the ideal sequence of $\mathcal{E}$ :

$$
0 \longrightarrow \mathcal{O}_{Y}(-\mathcal{E}) \longrightarrow \mathcal{O}_{Y} \longrightarrow \mathcal{O}_{\mathcal{E}} \longrightarrow 0
$$


Tensoring by $p^{*} \tau$ we get

$$
0 \longrightarrow p^{\prime *} \tau^{\prime} \longrightarrow p^{*} \tau \longrightarrow p^{*} \tau \otimes \mathcal{O}_{\mathcal{E}} \longrightarrow 0
$$

Let $\pi: Y \rightarrow X$ be the composition $\pi=f \circ p=f^{\prime} \circ p^{\prime}$. Consider the $\pi$ direct image of (A.2):

$$
0 \longrightarrow \pi_{*} p^{\prime *} \tau^{\prime} \longrightarrow \pi_{*} p^{*} \tau \longrightarrow \pi_{*}\left(p^{*} \tau \otimes \mathcal{O}_{\mathcal{E}}\right) \longrightarrow R^{1} \pi_{*} p^{\prime *} \tau^{\prime} \longrightarrow \ldots
$$

Observe first that every fiber of $\pi$ is either a projective space or has two irreducible components (meeting transversally) each of which is a projective space. Furthermore $p^{\prime *} \tau^{\prime}$ restricted on a component $P$ of the fiber is either $\mathcal{O}_{P}(1)$ or $\mathcal{O}_{P}$ and hence by Serre's vanishing theorem doesn't have higher cohomology. Thus by the base change and cohomology theorem $R^{1} \pi_{*} p^{\prime *} \tau^{\prime}=$ 0 . Next

$$
\pi_{*} p^{\prime *} \tau^{\prime}=f_{*}^{\prime} p_{*}^{\prime} p^{\prime *} \tau^{\prime}=f_{*}^{\prime} \tau^{\prime}=E^{\prime \vee}
$$

Here we used that $p^{\prime}: Y \longrightarrow \mathbb{P}\left(E^{\prime}\right)$ has connected fibers.

Similarly $\pi_{*} p^{*} \tau=E^{\vee}$ and we get

$$
0 \longrightarrow E^{\prime \vee} \longrightarrow E^{\vee} \longrightarrow \pi_{*}\left(p^{*} \tau \otimes \mathcal{O}_{\mathcal{E}}\right) \longrightarrow 0 .
$$

But $\pi_{*}\left(p^{*} \tau \otimes \mathcal{O}_{\mathcal{E}}\right)=\imath_{*} \bar{f}_{*}\left(\tau_{\mid \mathbb{P}(F)}\right)$ where $\bar{f}: \mathbb{P}(F) \longrightarrow D$ is the natural projection. Hence we get the short exact sequence

$$
0 \longrightarrow E^{\prime \vee} \longrightarrow E^{\vee} \longrightarrow \imath_{*} F^{\vee} \longrightarrow 0
$$

and thus

$$
E^{\prime}=\operatorname{Hecke}_{(\xi)}^{+}(E)
$$

\section{A.3 An example}

Let $X$ and $D$ be as before. One has the short exact sequence: 


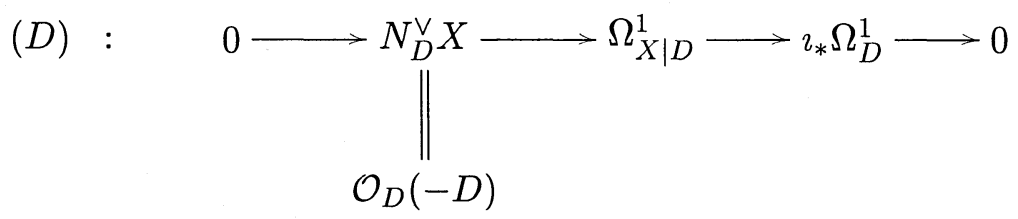

We can form the up and down Hecke transforms of $\Omega_{X}^{1}$ along $(D)$.

Lemma A.4. Denote by $\Omega_{X}^{1}(\log D)$ the sheaf of one forms on $X$ with logarithmic poles along $D$. Then the up and down Hecke transforms of $\Omega_{X}^{1}$ along (D) can be identified as follows

$$
\begin{aligned}
\boldsymbol{H e c k e}_{(D)}^{+}\left(\Omega_{X}^{1}\right) & =\Omega_{X}^{1}(\log D) \\
\boldsymbol{H e c k e}_{(D)}^{-}\left(\Omega_{X}^{1}\right) & =\Omega_{X}^{1}(\log D) \otimes \mathcal{O}_{X}(-D) .
\end{aligned}
$$

Proof. To prove the first equality observe that $\Omega_{X}^{1}(\log D)$ fits in the residue sequence

$$
0 \longrightarrow \Omega_{X}^{1} \longrightarrow \Omega_{X}^{1}(\log D) \longrightarrow \imath_{*} \mathcal{O}_{D} \longrightarrow 0,
$$

where the map $\Omega_{X}^{1}(\log D) \rightarrow \imath_{*} \mathcal{O}_{D}$ is given by the residue along $D$. On the other hand, according to Lemma A.2 we have an exact sequence

$$
0 \longrightarrow \Omega_{X}^{1} \longrightarrow \boldsymbol{H e c k e}_{(D)}^{+}\left(\Omega_{X}^{1}\right) \longrightarrow \imath_{*} \mathcal{O}_{D} \longrightarrow 0
$$

and it is easy to check that the two extension classes coincide.

\section{References}

[ACK] B. Andreas, G. Curio, and A. Klemm. Towards the Standard Model spectrum from elliptic Calabi-Yau, hep-th/9903052.

[BJPS97] M. Bershadsky, A. Johansen, T. Pantev, and V. Sadov. On four-dimensional compactifications of $F$-theory. Nuclear Phys. $B$, 505(1-2):165-201, 1997, hep-th/9701165.

[BM] T. Bridgeland and A. Maciocia. Fourier-Mukai transforms for K3 and elliptic fibrations, arXiv:math.AG/9908022. 
[DLOW99] R. Donagi, A. Lukas, B. Ovrut, and D. Waldram. Holomorphic vector bundles and non-perturbative vacua in M-theory. J. High Energy Phys., 1999(6):Paper 34, 46 pp. (electronic), 1999, hepth/9901009.

[Don97] R. Donagi. Principal bundles on elliptic fibrations. Asian J. Math., 1(2):214-223, 1997, alg-geom/9702002.

[DOPWa] R. Donagi, B. Ovrut, T. Pantev, and D. Waldram. Spectral involutions on rational elliptic surfaces, math.AG/0008011.

[DOPWb] R. Donagi, B. Ovrut, T. Pantev, and D. Waldram. StandardModel bundles on non-simply connected Calabi-Yau threefolds, hep-th/0008008.

[DOPWc] R. Donagi, B. Ovrut, T. Pantev, and D. Waldram. Standard models from heterotic M-theory, hep-th/9912208.

[DOW99] R. Donagi, B. Ovrut, and D. Waldram. Moduli spaces of fivebranes on elliptic Calabi-Yau threefolds. J. High Energy Phys., 1999(12):Paper 30, 58, 1999, hep-th/9904054.

[DPT80] M. Demazure, H.C. Pinkham, and B. Teissier, editors. Séminaire sur les Singularités des Surfaces, number 777 in Lecture Notes in Mathematics. Springer, 1980. Held at the Centre de Mathématiques de l'École Polytechnique, Palaiseau, 1976-1977.

[FMW97] R. Friedman, J. Morgan, and E. Witten. Vector bundles and F theory. Comm. Math. Phys., 187(3):679-743, 1997, hepth/9701162.

[FMW99] R. Friedman, J. Morgan, and E. Witten. Vector bundles over elliptic fibrations. J. Algebraic Geom., 8(2):279-401, 1999, alggeom/9709029.

[Fri98] R. Friedman. Algebraic surfaces and holomorphic vector bundles. Springer-Verlag, New York, 1998.

[Har77] R. Hartshorne. Algebraic geometry, volume 52 of Grad. Texts Math. Springer-Verlag, 1977.

[HW96a] P. Hořava and E. Witten. Eleven-dimensional supergravity on a manifold with boundary. Nuclear Phys. B, 475(1-2):94-114, 1996, hep-th/9603142.

[HW96b] P. Hořava and E. Witten. Heterotic and type I string dynamics from eleven dimensions. Nuclear Phys. B, 460(3):506-524, 1996, hep-th/9510209. 
[Kac95] S. Kachru. Some three generation $(0,2)$ Calabi-Yau models. Phys. Lett. B, 349(1-2):76-82, 1995, hep-th/9501131.

[Mar82] M. Maruyama. Elementary transformations in the theory of algebraic vector bundles. In Algebraic geometry (La Rábida, 1981), pages 241-266. Springer, Berlin, 1982.

[Mar87] M. Maruyama. On a generalization of elementary transformations of algebraic vector bundles. Rend. Sem. Mat. Univ. Politec. Torino, 1986(Special Issue):1-13, 1987. Conference on algebraic varieties of small dimension (Turin, 1985).

[Mum74] D. Mumford. Prym varieties. I. In Contributions to analysis (a collection of papers dedicated to Lipman Bers), pages 325-350. Academic Press, New York, 1974.

[PR99] W. Pokorski and G. Ross. Flat directions, string compactification and 3 generation models. Nucl.Phys. B, 551:515-548, 1999, hep-ph/9809537.

[Sch88] C. Schoen. On fiber products of rational elliptic surfaces with section. Math. Z., 197(2):177-199, 1988.

[Tho] R. P. Thomas. Examples of bundles on Calabi-Yau 3-folds for string theory compactifications, arxiv:math.AG/9912179.

[TY87] G. Tian and S.-T. Yau. Three-dimensional algebraic manifolds with $C_{1}=0$ and $\chi=-6$. In Mathematical aspects of string theory (San Diego, Calif., 1986), pages 543-559. World Sci. Publishing, Singapore, 1987.

[UY86] K. Uhlenbeck and S.-T. Yau. On the existence of HermitianYang-Mills connections in stable vector bundles. Comm. Pure Appl. Math., 39(S, suppl.):S257-S293, 1986. Frontiers of the mathematical sciences: 1985 (New York, 1985).

[Wit96] E. Witten. Strong coupling expansion of Calabi-Yau compactification. Nuclear Phys. B, 471(1-2):135-158, 1996, hepth/9602070. 